\title{
3D inversion and resolution
}

analysis of land-based CSEM

data from the Ketzin CO 2 storage

\section{formation}

\section{Journal Article}

Author(s):

Grayver, Alexander; Streich, Rita; Ritter, Oliver

Publication date:

2014-03

Permanent link:

https://doi.org/10.3929/ethz-b-000109205

Rights / license:

In Copyright - Non-Commercial Use Permitted

Originally published in:

GEOPHYSICS 79(2), https://doi.org/10.1190/geo2013-0184.1 


\title{
3D inversion and resolution analysis of land-based CSEM data from the Ketzin $\mathrm{CO}_{2}$ storage formation
}

\author{
Alexander V. Grayver ${ }^{1}$, Rita Streich ${ }^{2}$, and Oliver Ritter ${ }^{1}$
}

\begin{abstract}
We evaluated 3D inversion of land controlled-source electromagnetic (CSEM) data collected across the Ketzin $\mathrm{CO}_{2}$ storage formation. A newly developed, parallel and distributed 3D inversion code, which is based on a direct forward solver, has been used. This inversion scheme allowed us to calculate the Jacobian matrix explicitly within a reasonable time and use it to calculate regularization parameters, inspect survey coverage, and carry out resolution analysis. After demonstrating that the magnetic field components are sensitive to conductors only, whereas the electric field components are sensitive to all features of interest, we continued to work with electric field data only. Estimates of data uncertainty obtained from robust processing were used for automated data preselection and weighting during
\end{abstract}

inversion. We tested different regularization techniques and a range of starting models to explore the model space and assess the influence of regularization on the inversion images. We further demonstrated an approach for handling numerical singularities due to sources located inside the inversion domain. We estimated survey coverage, horizontal and vertical resolution, and depth penetration using cumulative sensitivity, point spread functions, and depth-resolution plots. Based on data fit analysis, we determined a preferred subsurface conductivity model, which we compared to an independent regional structural geologic model, and we provided an interpretation for the structures resolved. The inversion approach we used provides robust results in good agreement with known geology, offers new possibilities for model assessment, and should be transferable to other CSEM data sets.

\section{INTRODUCTION}

To date, controlled-source electromagnetic (CSEM) in the frequency domain is predominantly used for offshore (Constable, 2010) and airborne (Siemon et al., 2009) studies in the industrial and academic domains. Whereas airborne configurations necessitate inductive source excitation, present-day marine CSEM (mCSEM) most commonly uses horizontal bipole sources galvanically coupled to the sea (Constable, 2013). A horizontal electric source generates horizontal and vertical current flow, thus facilitating the imaging of conductive and resistive targets.

Analogous applications of frequency-domain CSEM with galvanic sources on land have rarely been reported, although feasibility studies exist (e.g., Wirianto et al., 2010). This is likely due to a variety of reasons, including difficulty in deploying sources of sufficient strength, weakening of subsurface responses compared to marine CSEM responses by the signal traveling through air (Constable, 2010), frequent occurrence of near-surface inhomogeneity complicating data interpretation, and the necessity to operate at relatively large source-receiver distances where signal levels are low and abundant noise may severely distort the data.

Many previous land EM applications interpreted the data in the time domain (e.g., Keller et al., 1984; Hördt et al., 2000; Kalscheuer et al., 2007) and used magnetic dipole sources (Kurtz et al., 1989; Mitsuhata et al., 2006; Minsley et al., 2012; Perez-Flores et al., 2012). Unfortunately, standard transient EM (TEM) configurations with magnetic dipole sources have limited capability of resolving resistors (Chave, 2009). At the same time, the time and frequency domains are equivalent in theory, being related through a unique transform. Recent work demonstrates similar resolution properties for time- and frequency-domain EM methods in marine and airborne configurations (Yang and Oldenburg,

Manuscript received by the Editor 13 May 2013; revised manuscript received 23 August 2013; published online 3 March 2014.

${ }^{1}$ GFZ German Research Centre for Geosciences, Telegrafenberg, Potsdam, Germany. E-mail: agrayver@gfz-potsdam.de; oritter@gfz-potsdam.de.

${ }^{2}$ Formerly GFZ German Research Centre for Geosciences, Telegrafenberg, Potsdam, Germany; presently Shell Global Solutions International BV, Rijswijk, The Netherlands. E-mail: rita.streich@shell.com.

(C) 2014 Society of Exploration Geophysicists. All rights reserved. 
2012; Connell and Key, 2013). Limitations of TEM for imaging resistors and inspection of theoretical resolution capabilities encourage us to reconsider frequency-domain land-based CSEM with grounded sources.

The recent upsurge in mCSEM applications for hydrocarbon exploration has led to intensive research and knowledge generation, including insights from synthetic studies (Weiss and Constable, 2006; Um and Alumbaugh, 2007; Commer and Newman, 2008; Sasaki and Meju, 2009) as well as real data inversions (e.g., Newman et al., 2010; Schwalenberg et al., 2010; Li et al., 2011; Weitemeyer et al., 2011; Súilleabháin et al., 2012). Whereas the physics of the method is the same for surveys on land, inversion of land CSEM data poses several challenges not equally encountered in mCSEM. Source-receiver coverage is usually sparser than for mCSEM studies because arbitrary deployment of long-wire transmitters and receivers on land is logistically more difficult and typically restricted in populated areas with abundant infrastructure. In addition, whereas treating the sources as unit dipoles is adequate for many mCSEM surveys, kilometer-long sources cannot be approximated by idealized unit dipoles without introducing significant errors (Streich and Becken, 2011). Therefore, we need to handle real source geometries accurately while keeping run times reasonable. Furthermore, whereas water conductivity can be measured in situ and kept fixed for many mCSEM inversions, grounded land transmitters are located inside the portion of the model being updated during inversion. Numerical singularities arise in model cells that contain parts of a transmitter. Therefore, special care is required to prevent severe artifacts in these regions, which may otherwise corrupt the inversion.

This work is a practical application study of 3D land CSEM inversion. We begin with a brief description of the inversion algorithm applied (Grayver et al., 2013). The field experiment carried out across the Ketzin $\mathrm{CO}_{2}$ storage formation is then described. Based on the field survey layout and noise conditions, we select a nonredundant data subset for inversion. We then present 3D inversions of the real data and discuss technical aspects that were crucial for obtaining models that reasonably explain the observed data. Specifically, we focus on real source handling, the definition of starting models, and the effects of different regularization techniques. Based on these studies and the overall data fit, we select a preferred model. For this model, we present detailed analyses of data fit and cumulative sensitivity, and we estimate spatial resolution using point spread functions (PSFs) and depth penetration using depth-resolution plots. Finally, we compare the preferred resistivity model with an independent regional structural geologic model.

\section{FORWARD MODELING}

The forward solver used in this study is based on a finitedifference approximation of the vector Helmholtz equation for the electric field (Streich [2009], equation 3). We solve this equation using a direct solver. This provides robustness and efficiency for multiple transmitters and Jacobian matrix calculations (Grayver, 2013). To model realistic source geometries accurately without using very fine meshes in the vicinity of the source, we exploit a secondary-field approach (Newman and Alumbaugh, 1995).

\section{INVERSION ALGORITHM}

We minimize the nonlinear functional (Menke, 1984)

$$
\phi(\mathbf{m})=\frac{1}{2} \phi_{d}(\mathbf{m})+\frac{\beta}{2} \phi_{m}(\mathbf{m}) .
$$

The term $\phi_{d}(\mathbf{m})$ represents the data misfit in the form of the weighted $L_{2}$-norm

$$
\phi_{d}(\mathbf{m})=\left(\mathbf{f}(\mathbf{m})-\mathbf{d}^{\mathrm{obs}}\right)^{H} \mathbf{W}_{d}^{T} \mathbf{W}_{d}\left(\mathbf{f}(\mathbf{m})-\mathbf{d}^{\mathrm{obs}}\right),
$$

where vectors $\mathbf{m} \in \mathcal{R}^{N_{m}}$ and $\mathbf{d}^{\text {obs }} \in \mathcal{C}^{N_{d}}$ represent the model parameters (in this case, electric resistivities) and measured data, respectively; scalars $N_{m}$ and $N_{d}$ denote the numbers of model parameters and complex data values; and $\mathbf{f}(\mathbf{m}): \mathcal{R}^{N_{m}} \rightarrow \mathcal{C}^{N_{d}}$ is the forward operator. The superscript $H$ denotes conjugate transpose. The diagonal weighting matrix $\mathbf{W}_{d} \in \mathcal{R}^{N_{d} \times N_{d}}$ is given by

$$
\mathbf{W}_{d}=\operatorname{diag}\left(\frac{1}{\left|d_{i}^{\mathrm{obs}}\right| r_{i}+\varepsilon}\right) ; \quad i=1, \ldots, N_{d},
$$

where estimates of data errors $r_{i}$ are obtained from data processing and expressed as fractions of data amplitude and $\varepsilon=10^{-15}$ is the absolute error floor.

The stabilizing functional $\phi_{m}(\mathbf{m})$ (Tikhonov and Arsenin, 1977; Zhdanov, 2002) in equation 1 regularizes the ill-posed inverse problem and constrains the solution. The regularization parameter $\beta \geq$ $0 \in \mathcal{R}$ controls the influence of the regularization term.

Applying a second-order Taylor expansion to equation 1 and dropping second-order derivative terms results in the Gauss-Newton approximation of the expansion. Enforcing the necessary condition that the first derivative is zero at local minima then allows us to write the system of normal equations:

$$
\left(\operatorname{Re}\left\{\overline{\mathbf{J}}^{H} \overline{\mathbf{J}}\right\}+\beta \mathbf{K}\right) \delta \mathbf{m}=-\left(\operatorname{Re}\left\{\overline{\mathbf{J}}^{H} \delta \mathbf{d}\right\}+\beta \mathbf{K}\left(\mathbf{m}-\mathbf{m}^{\text {ref }}\right)\right) .
$$

Here, $\delta \mathbf{m} \in \mathcal{R}^{N_{m}}$ is a model update vector, $\overline{\mathbf{J}}=\mathbf{W}_{d} \mathbf{J} \in \mathcal{C}^{N_{d} \times N_{m}}$ is the weighted sensitivity matrix, and $\delta \mathbf{d}=\mathbf{W}_{d}\left(\mathbf{f}(\mathbf{m})-\mathbf{d}^{\text {obs }}\right)$ is the weighted data residuals. The regularization matrix $\mathbf{K}$ is a discrete representation of the stabilizing functional. In this work, we use $\mathbf{K}=\mathbf{L}^{T} \mathbf{L}$ with the matrix $\mathbf{L}$ being a 3D finite-difference approximation of the Laplacian and $\mathbf{K}=\mathbf{I}$, which provides a minimumnorm solution (Zhdanov, 2002). The matrix on the left side of equation 4 is the regularized approximate Hessian. The right side is the negative gradient representing the descent direction.

We solve the system of normal equation 4 using a conjugate gradient (CG) algorithm (Golub and van Loan, 1996). A new model is derived as the sum of the current model and a scaled model update vector obtained from equation 4

$$
\mathbf{m}_{n+1}=\mathbf{m}_{n}+\alpha \delta \mathbf{m}_{n},
$$

where the step length $\alpha$ controls the magnitude of the model update. A suitable step length is determined using a line-search algorithm (Nocedal and Wright, 1999). To ensure that the model parameters stay within physically reasonable limits, we use bounded transformations of the conductivity as described in Kim and Kim (2011).

We have found that a reasonable starting guess for the regularization parameter can be calculated using 


$$
\beta=\frac{\gamma \beta_{L_{2}}}{n_{\text {iter }}}
$$

where $\gamma \in[0,1]$. The division by the iteration count $n_{\text {iter }}$ implements a cooling approach (Haber et al., 2000). The scaling factor $\beta_{L_{2}}$ is recalculated at each inversion iteration as (Grayver et al., 2013; Schwarzbach and Haber, 2013)

$$
\beta_{L_{2}}=\frac{\left\|\operatorname{Re}\left\{\overline{\mathbf{J}}^{H} \overline{\mathbf{J}}\right\}\right\|_{2}}{\|\mathbf{K}\|_{2}}
$$

We can calculate the $L_{2}$-norms of these matrices efficiently because we precompute the Jacobian and sparse regularization matrices (Grayver et al., 2013). The scaling factor $\beta_{L_{2}}$ weights the dataand regularization-related terms equally and represents a practical upper bound for the regularization parameter.

The inversion scheme has been implemented in a fully distributed manner to run efficiently on modern computational platforms. The code extensively uses the PETSc library (Balay et al., 2012) for distributed linear algebra. Its interface to the distributed direct solver MUMPS (Amestoy et al., 2006) is used in the forward

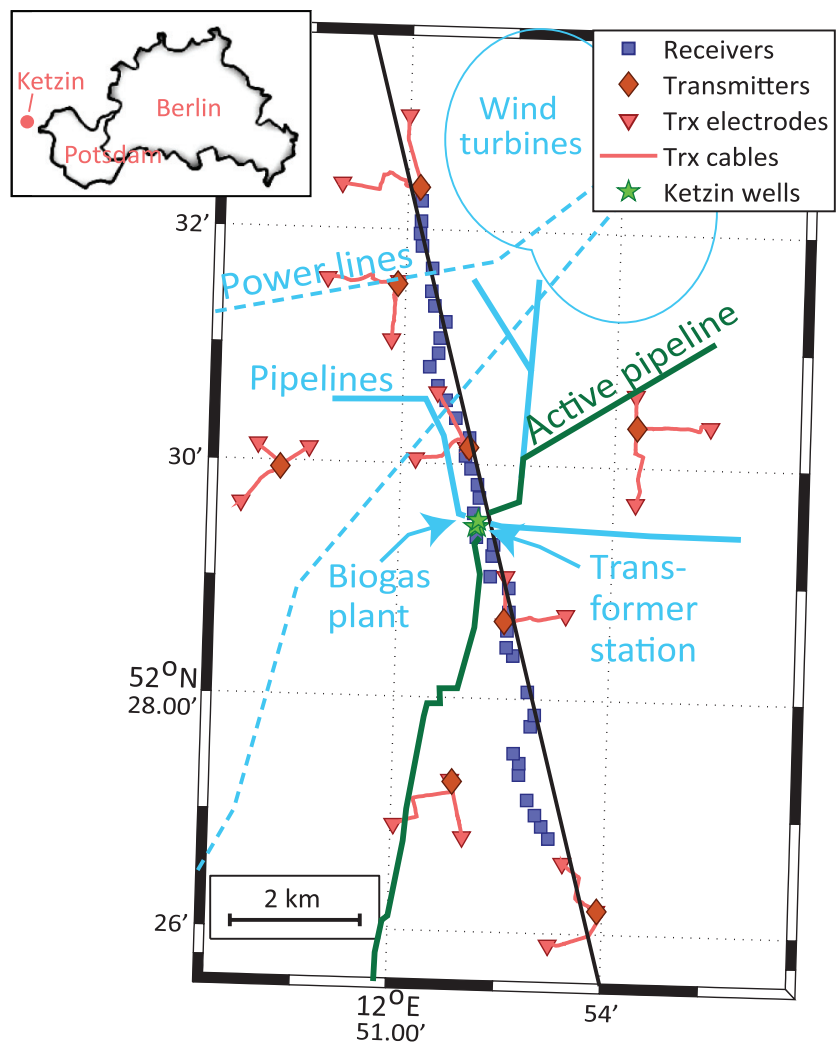

Figure 1. Location of the Ketzin survey site and layout of the CSEM survey. Squares denote receivers. Red lines, triangles, and diamonds indicate transmitter cables, grounded electrodes and generator locations. Light blue features show approximate locations of major sources of cultural noise. The green line indicates a pipeline on which pulsed anticorrosion currents were present. The black line indicates the location at which sections are extracted from inversion models; the labels "Distance" in subsequent figures refer to distance along this line. modeling. Furthermore, we use model domain decomposition to parallelize the primary field calculations. We have also parallelized the code over frequencies because the solutions for different frequencies can be obtained independently. This multilevel parallelization ensures that the workload is distributed evenly over all $N_{\text {proc }}$ processes used, and each process only requires memory for approximately the $1 / N_{\text {proc }}$ th part of any allocated matrix or vector.

\section{FIELD EXPERIMENT}

A land CSEM survey was carried out across the $\mathrm{CO}_{2}$ storage formation at Ketzin, Germany. The location of the survey area and acquisition geometry are shown in Figure 1. For the remainder of the paper, we adopt a local coordinate system with the $x$ - and $y$-axes aligned with geographic north and east, respectively. The origin of the local coordinate system is located at the bottom-left corner of the box displayed in Figure 1. Eight CSEM transmitters and 39 five-component receivers were deployed along an 11-kmlong receiver line centered at the $\mathrm{CO}_{2}$ injection site. The regional geology is generally well studied (e.g., Förster et al., 2006), rendering it well-suited for testing newly developed hardware and imaging tools such as 3D CSEM. Altitude differences throughout the survey area do not exceed $30 \mathrm{~m}$. Therefore, we can reasonably assume flat topography in our inversions and avoid computationally expensive and numerically difficult handling of topography (Commer et al., 2005). Unfortunately, the site is located in a populated region with abundant infrastructure. Nearby railways, major power lines, an array of wind turbines, and a gas pipeline that carried pulsed anticorrosion currents and crossed the receiver line at $y \approx 7 \mathrm{~km}$ (see Figure 1), all caused strong cultural noise and distorted the CSEM data. We used strong currents up to $40 \mathrm{~A}$ and relatively long operation times of $12-14 \mathrm{~h}$ per transmitter to obtain adequate signal-tonoise ratios.

The transmitter used in the survey (Figure 2) simultaneously injects currents through three grounded electrodes (Streich et al., 2013). The three currents are determined as

$$
I_{i}=I(\omega) \cos \left(120^{\circ}(i-1)+\psi\right) ; \quad i \in(1,2,3),
$$

where $I(\omega)$ is a predefined waveform and the angle $\psi$ can be adjusted during operation of the transmitter. The constant $120^{\circ}$ phase

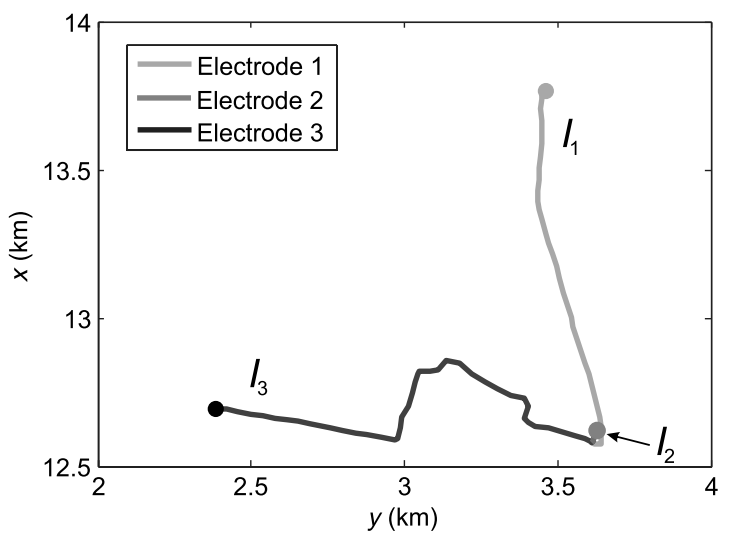

Figure 2. Enlarged view of transmitter Trx06 (see Figure 1) with the three current-carrying source wires shown in grayscale. 
shifts between the three current waveforms imply that the sum of the currents is zero at all times. Therefore, we can write any field component $F$ in the frequency domain in terms of two source currents as

$$
F=\left(\begin{array}{ll}
T_{13}^{F} & T_{23}^{F}
\end{array}\right)\left(\begin{array}{c}
I_{1} \\
I_{2}
\end{array}\right)
$$

Here, $I_{1}$ and $I_{2}$ can be any two of the source currents and $T_{13}^{F}=$ $T_{1}^{F}-T_{3}^{F}$ and $T_{23}^{F}=T_{2}^{F}-T_{3}^{F}$ are the corresponding response functions (for brevity, the frequency dependency of all quantities is omitted). These response functions contain the desired information on subsurface resistivity but also depend on the source geometry. Response functions and their uncertainties have been obtained from statistically robust processing.

\section{DATA SELECTION}

The collected data set contains five EM field components within the frequency range of 1/64-150 Hz. For each field component,
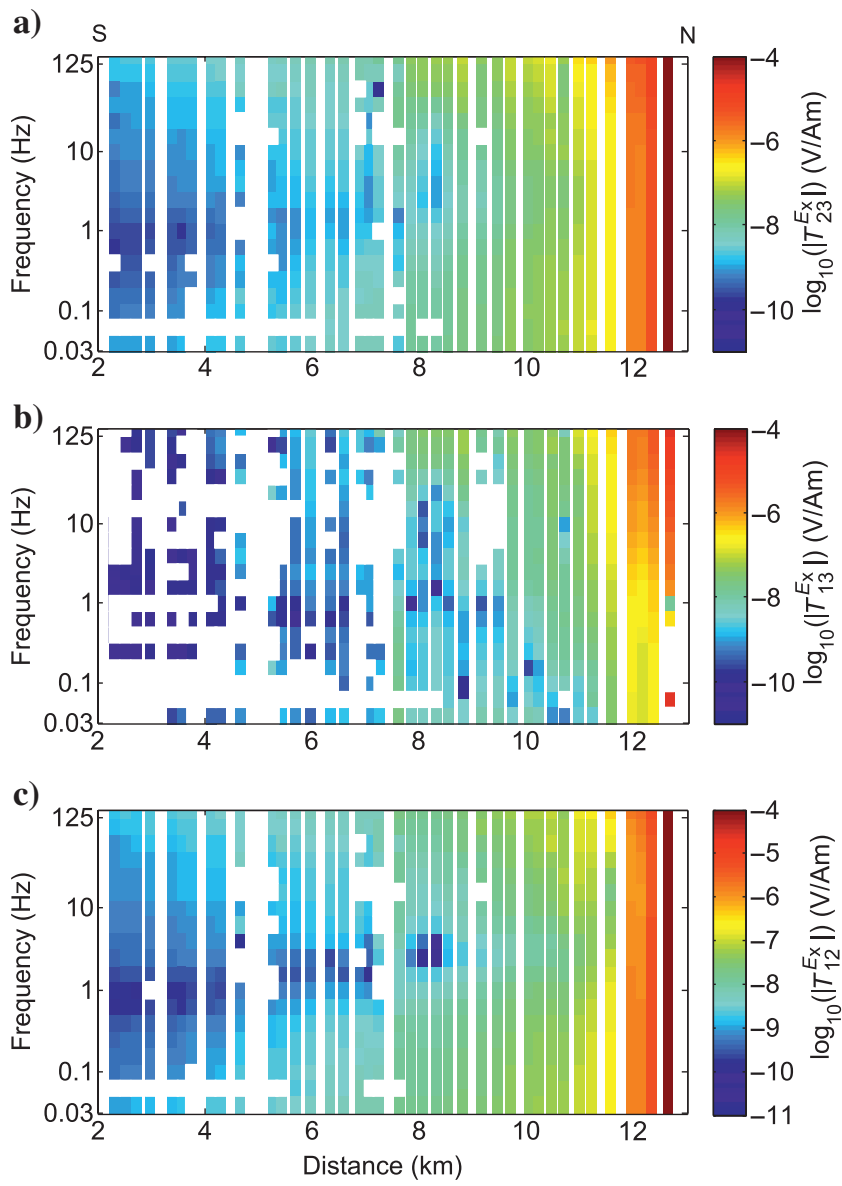

Figure 3. Amplitudes of the three response functions (a) $T_{23}^{F}$, (b) $T_{13}^{F}$, and (c) $T_{12}^{F}$ derived for field component $E_{x}$ and transmitter Trx06 (see Figure 1). Each vertical column represents data for one receiver. Data with estimated uncertainties larger than $12 \%$ are not shown. The response function $T_{13}^{F}$ (b) is noisier than the other two. The horizontal axis shows distance along the black line depicted in Figure 1. three different response functions can be determined, corresponding to different sets of source currents in equation 9. The entire data set then comprises approximately 90,000 complex data values at 20 different frequencies. Parts of the collected data are redundant because of overlapping information in different frequencies and EM field components and as a result of the transmitter setup with linearly dependent currents (Grayver, 2013). Redundant data enlarge the null space of the Jacobian matrix; including redundant data in inversions requires additional computational resources without improving the solution (Jupp and Vozoff, 1975). To run 3D inversion efficiently, we aim to find a nonredundant data subset that effectively contains all of the subsurface information and allows us to run multiple inversions within manageable time.

Because only two of the three response functions we obtain from the data are linearly independent, we can discard one of them. For each transmitter and field component, we discard one response function at all receivers and frequencies. We anticipate that this preserves some important yet somewhat noisy low-amplitude features better than point-by-point selection of response functions. To determine which response to discard, we count the number of data points with estimated uncertainty higher than $12 \%$ of the data amplitude. Then, the response with the largest number of such poorly determined data values is dropped. The threshold of $12 \%$ represents the standard deviation of the estimated uncertainties.

Examples of the three response functions for transmitter Trx06 and all receivers are shown in Figure 3 . The response $T_{13}^{F}$ generally has the lowest amplitudes and the largest number of noisy data values. For the source-receiver geometry used, Ex fields due to electrodes 1 and 3 (depicted in Figure 2) of this transmitter partially cancel out each other. This results in low data amplitudes for $T_{13}^{E x}\left(=T_{1}^{E x}-T_{3}^{E x}\right)$, which are difficult to determine precisely because of the high level of cultural noise in the survey area.

Furthermore, we select five frequencies at approximately logarithmically uniform spacing, namely, 0.0622, 0.156, 0.392, 0.986 , and $6.22 \mathrm{~Hz}$. This frequency range is significantly wider than ranges commonly used in mCSEM inversion (Commer and Newman, 2008; Weitemeyer et al., 2010), which may partially compensate for the sparser land survey geometry. Synthetic and real data tests showed that denser frequency spacing or additional use of higher frequencies did not result in more detailed images of subsurface structure.

Magnetic fields generally have little sensitivity to resistive objects (e.g., Oldenburg et al., 2013). In contrast, electric field components are sensitive to resistors and conductors (e.g., Weiss and Constable, 2006). To demonstrate sensitivities of the different field components to the expected subsurface structure, we run synthetic inversions using the real acquisition geometry. We embed resistive and conductive blocks of 50 and $1 \Omega \mathrm{m}$ in a homogeneous halfspace of $5 \Omega \mathrm{m}$. The blocks have dimensions of $3.0 \times 2.5 \times$ $0.1 \mathrm{~km}$ and are located in the center of the model with their tops at a depth of $0.6 \mathrm{~km}$ (Figure $4 \mathrm{a}$ and $4 \mathrm{~d}$ ). We used three frequencies of $0.1,1$, and $10 \mathrm{~Hz}$, which cover a similar range as the frequencies extracted from the field data. Two percent random noise is added to the synthetic data generated for these models.

For each model, we separately invert the horizontal electric and magnetic field components. We use data for transmitter-receiver distances larger than $700 \mathrm{~m}$, resulting in 5472 complex data values. The inversion domain is limited to the subsurface region, excluding boundary cells added to ensure the validity of boundary conditions 
in forward modeling. The data do not constrain conductivity in the boundary cells. We found that excluding these cells virtually did not influence the inversion results in the region of interest while reducing the size of the inversion problem. With data error $r_{i}$ (see equation 3 ) defined as $2 \%$ of the data amplitudes, all inversions fit the data equally well within the noise level. As expected, the magnetic field components are not sensitive to the resistive block (Figure $4 \mathrm{~b}$ and $4 \mathrm{c}$ ), whereas the electric field components resolve the resistor reasonably well. The conductive block is resolved by the electric and magnetic fields and is better focused in the image obtained from the electric field components (Figure $4 \mathrm{e}$ and $4 \mathrm{f}$ ). This test indicates that the magnetic field data are unlikely to add subsurface information to that contained in the electric field data. This observation was confirmed when we tested using real magnetic field data in the inversion. Therefore, we only include the horizontal electric field components in the real data inversion.

Finally, based on inspection of data uncertainty levels and data consistency along the receiver line and over frequencies, we drop data with estimated uncertainties higher than $12 \%$. This resulted in rejection of approximately $18 \%$ of the data values. We also drop data for all receivers located within $700 \mathrm{~m}$ of any of the source electrode positions because near-source data are strongly influenced by local near-surface inhomogeneities and have little sensitivity to deeper structure. The resulting data subset used in the inversion contains 3957 complex data values. The data error estimations obtained during data processing are used to weight the data in the inversion. Because there is no rigorous criterion for distinguishing noise from data, we refrain from manually masking any data points beyond the selection procedure described to avoid introducing possible bias. To preserve information on uncertainty in the data weighting matrix (equation 3), we use an error floor of $2 \%$ (i.e., $r_{i}=$ $\max \left(0.02, r_{i}^{\text {proc }}\right)$ for processing error estimates $\left.r_{i}^{\text {proc }}\right)$.

\section{INVERSION OF REAL DATA}

\section{Discretization}

We define an inversion domain of $15 \times 10 \times 2.8 \mathrm{~km}$ that includes all transmitters and receivers. The inversion domain is discretized using a grid of $40 \times 40 \times 40$ cells with uniform $x$ and $y$ cell sizes of $375 \times 250 \mathrm{~m}$. In the $z$-direction, we use one cell of 5-m thickness at the surface. Subsequent cells grow in size up to $50 \mathrm{~m}$, and a constant size of $50 \mathrm{~m}$ is used at depths below $200 \mathrm{~m}$. At the top of the model, an air layer having a resistivity of $10^{9} \Omega \mathrm{m}$ is added. To prevent boundary artifacts in forward modeling, the inversion domain is augmented by padding cells that grow in size at a factor of 1.5. To determine the necessary number of padding cells, we successively augmented the inversion domain until the calculated responses remained constant, indicating that the boundary conditions were sufficiently fulfilled. The final grid, including padding cells, has a size of $30 \times 34 \times 25 \mathrm{~km}$ and consists of $50 \times 54 \times 80$ cells.

To verify the quality of the grid, we compare a 3D numerical solution to a 1D quasianalytical solution for the layered model shown in Figure 5a, which represents the anticipated resis- tivity structure. The background model for 3D forward modeling was a homogeneous half-space of $5 \Omega \mathrm{m}$. A histogram of amplitude ratios between numerical and quasianalytical solutions for the horizontal electric field components is shown in Figure 5b. Although $73 \%$ of the data values obtained from 3D modeling are within $\pm 2 \%$ of the quasianalytical results, some values have significantly larger errors. To analyze the errors further, we display in Figure 6 horizontal electric fields and amplitude ratios for all transmitters at a frequency of $0.98 \mathrm{~Hz}$. As expected, the largest errors occur near the transmitters, where the EM field exhibits particularly strong variations that cannot be captured accurately within the used discretization. Nevertheless, because we drop the data points within $700 \mathrm{~m}$ from any of the transmitter electrodes, most of these inaccuracies do not affect our data. This is shown in the histogram in Figure $5 c$ that excludes data near the transmitters and values for which uncertainties of the real data exceed $12 \%$; this corresponds exactly to the data subset used in the inversion. For this subset, $78.4 \%$ of the data have errors less than $\pm 2 \%$ and $88.7 \%$ of the data have errors less than $\pm 3 \%$. The overall mean percentage difference is $2.2 \%$. Similar results have previously been reported in Weitemeyer et al. (2010).

Increasing the widths of the boundary regions did not result in more accurate modeling results. The accuracy could be improved further by refining the grid (Figure 5d). This, however, would increase the number of unknowns. Given the same amount of data, the inversion would then become more ambiguous and less stable. To overcome this trade-off and achieve highly accurate forward modeling when inverting data sets that require coarse inversion grids, we are considering separating the forward modeling and inversion grids (Commer and Newman, 2008).

\section{Inversion parametrization}

Unless otherwise stated, we use smoothing regularization and run $500 \mathrm{CG}$ iterations to solve equation 4. Additionally, the CG solver is terminated if the normalized relative residual reaches a value of $10^{-3}$. All inversions were terminated after 30 Gauss-Newton iterations. The discrete Laplacian matrix, having entries proportional to $1 / d \alpha^{2} ; \alpha=x, y, z$, imposes relatively strong smoothing in the a)

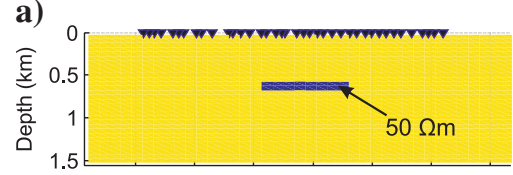

b)

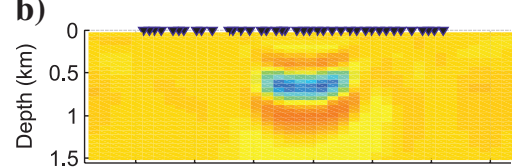

c)

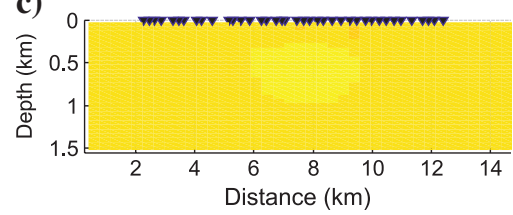

d)

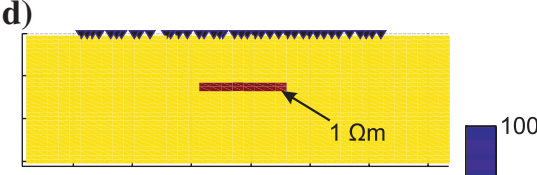

e)

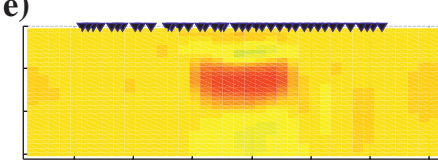

f)

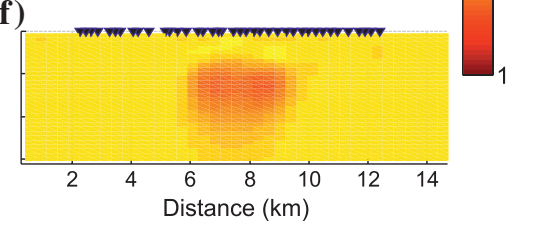

Figure 4. Synthetic models containing (a) resistive and (d) conductive blocks. Black triangles indicate receivers. Sections were extracted from 3D inversion models along the profile line marked in Figure 1. Inversions results are shown for (b and e) the electric field components $E_{x}$ and $E_{y}$, and (c and f) the magnetic field components $H_{x}$ and $H_{y}$. 
vertical direction as a result of the smaller vertical cell sizes. Therefore, we used a vertical to horizontal smoothing ratio of 1:5 to achieve similar smoothing in the vertical and horizontal directions. Depending on the stabilizing functional and its spectrum, different ranges of suitable values for $\gamma$ in equation 6 can be inferred. For smoothing regularization, we found that for many synthetic and real inversions, $\gamma \in[0.01,0.1]$ provides reasonable results (Grayver et al., 2013). Therefore, we used $\gamma=0.04$ in this work (for a test with minimum-norm regularization, $\gamma=10^{-4}$ was used). For all inversions shown, we constrained resistivity to the interval $0.2<\rho<10000 \Omega \mathrm{m}$.

\section{Starting model}

The inversion based on the Gauss-Newton minimization method searches for a local minimum given an initial position in the objective functional space. Therefore, inversions should be run with a)

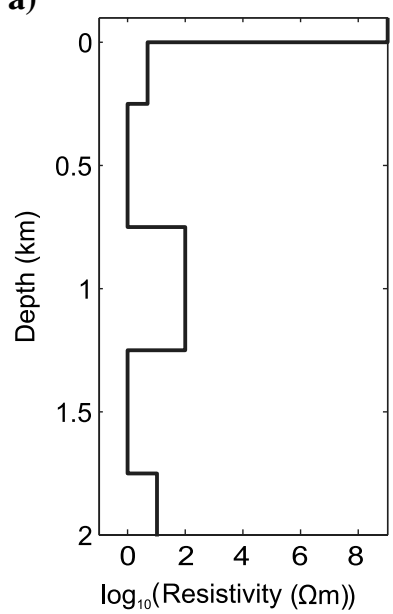

c)

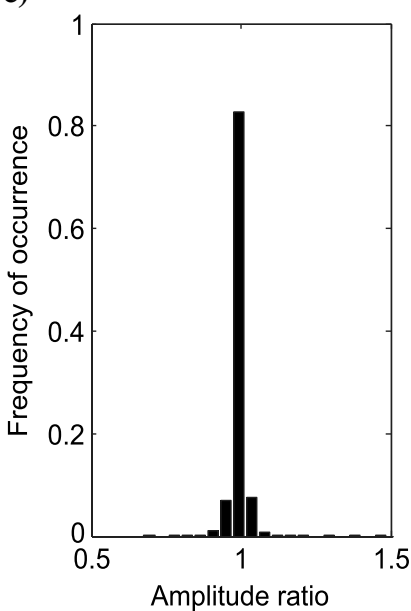

b)

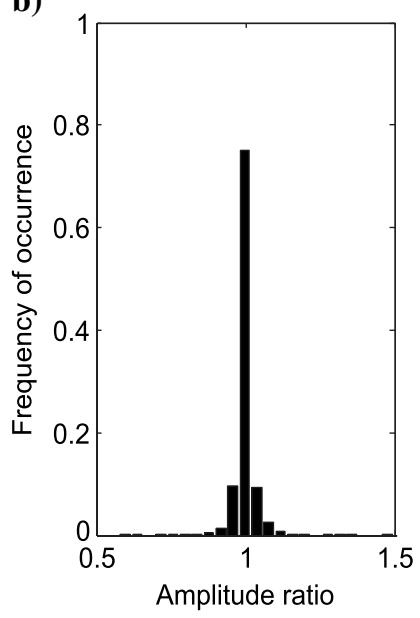

d)

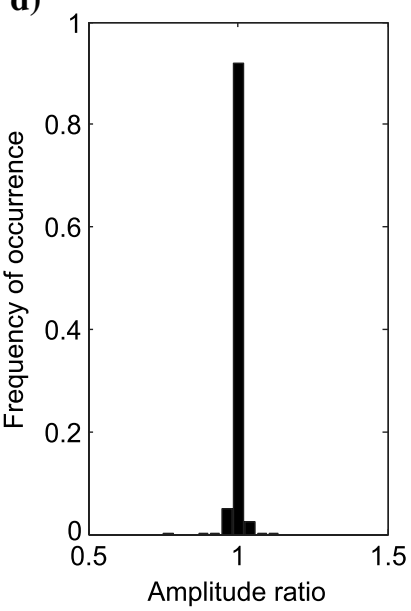

Figure 5. (a) Layered model used for calculating quasianalytical 1D and numerical 3D solutions. (b) Histogram of the amplitude ratios between numerical and quasianalytical solutions. (c) Same as (b), but excluding data values within $700 \mathrm{~m}$ from any of the source electrodes. (d) Same as (c), but calculated for a modeling grid with the $x$ and $y$ cell sizes halved. different starting models to investigate the model space and the stability of the obtained results.

We tested a range of homogeneous half-space starting models of different resistivity. Figure $7 b-7 d$ shows inversion results obtained by starting from homogeneous half-spaces of 1,5 , and $20 \Omega \mathrm{m}$, respectively. In what follows, we will provide root-mean-square (rms) values for the models shown. The rms is calculated as

$$
\mathrm{rms}=\sqrt{\frac{\phi_{d}(\mathbf{m})}{N_{d}}} .
$$

Notably, the lowest rms of 12.9 is achieved for starting from a $1 \Omega \mathrm{m}$ half-space. The image, however, appears inconsistent and noisy. The image obtained for a starting half-space model of $20 \Omega \mathrm{m}$ has a significantly higher final $\mathrm{rms}$ of 23.3 . The image obtained for starting from a $5 \Omega \mathrm{m}$ half-space has a relatively low rms of 13.5.

In addition, we tested a starting model obtained from a set of 1D inversion results (Figure 7e). Gauss-Newton-type 1D inversions were carried out using a different data subset containing all four horizontal EM field components and 20 frequencies. A homogeneous $7-\Omega m$ half-space was used as the starting model. This value was chosen prior to detailed investigation of optimum starting resistivity for $3 \mathrm{D}$ inversion. The $1 \mathrm{D}$ inversion results for most transmitter-receiver pairs were robust against moderate changes of the starting model. Therefore, we expect that our findings regarding a 1D-inversion-based starting model would not change strongly if we used the starting value of $5 \Omega \mathrm{m}$ that was later found to be optimal for $3 \mathrm{D}$ inversion. The individual result obtained for each transmitter-receiver pair was projected to the center between the transmitter and receiver. Then, for each horizontal slice, we interpolated the $1 \mathrm{D}$ results onto the inversion grid using 2D kriging (Cressie, 1993). A section of the interpolated resistivities along the profile line is shown in Figure 7a. The image resulting from the starting model derived from 1D inversions is similar to that obtained for a $5 \Omega \mathrm{m}$ half-space, but it has a slightly higher rms of 14.9 and retains high-resistivity extrapolation artifacts near the ends of the survey line. We, therefore, prefer a homogeneous half-space of $5 \Omega \mathrm{m}$ as the starting model in this study.

\section{Model updates at source locations}

We use a secondary field approach that allows us to model realistic sources of complex geometry accurately without having to use very fine grids near the sources. Using this approach, secondary sources arise in cells whose conductivity differs from the background conductivity. In what follows, we calculate 1D layered background models by averaging conductivities over each horizontal slice of the starting model (i.e., when starting from a half-space, our background model also is a half-space). For these 1D models, we calculate the primary EM fields quasianalytically.

In the field survey, we used long-wire sources up to $2 \mathrm{~km}$ long grounded through steel electrodes extending to depths of 5-10 m (depending on local coupling resistance) and copper wires laid out at the surface as shown in Figure 1. The size of these sources requires taking into account the real source geometry. To do this, we separately calculate and add the contributions from the grounded electrodes and the inductive component from the wires. This is equivalent to, yet slightly more efficient than, representing the 
source as a set of point dipoles following the source trajectory (Streich and Becken, 2011). For further efficiency gain, we place the entire sources slightly below the air-ground interface in the modeling grid; this is permissible because variations in the inductive component for wires placed immediately above and below the surface are minor. This allows us to capture the contributions from the grounded electrodes accurately. If the model is updated during inversion and the background model remains fixed, secondary sources arise in the cells being crossed by the physical sources. This introduces numerical singularities in these cells, which can result in severe numerical artifacts corrupting the inversion. Therefore, we need to ensure that the conductivity of the cells intersected by sources equals the background model conductivity whenever a forward solution is required. Because the topography in our model is flat, all sources are located within the same horizontal layer.

We test two ways of preventing source singularities in forward modeling. First, we fix the resistivity of the layer containing sources and test a range of thicknesses for this layer. Second, we shift the sources slightly into the air. The horizontal electric field components we invert are continuous across the air-ground interface, and shifting the sources does not change responses strongly.

Figure $8 \mathrm{a}-8 \mathrm{c}$ shows results obtained with the first subsurface layer being fixed during inversion. Clearly, a 2-m-thin layer results in an inhomogeneous model. This indicates that the singularity removal is not sufficient; we have strong secondary sources very close to the physical sources. Using a 40-m-thick layer of fixed resistivity results in a much higher rms of 20.8 and more resistive structure throughout the volume. Likely, the inversion is unable to effectively compensate for near-surface inhomogeneities required to fit the data. Fixing a 5-m layer provides an image with consistent structures and a low rms value of 13.5. For the sources shifted into the air, we did not achieve good data fit, and the models differ significantly from those obtained for the sources in the subsurface. Therefore, we run further inversions using a 5-m-thick layer that was fixed to that of the starting background model. We realize that the resistivity assigned to this layer influences the inversion results. Tests of updating this layer by recalculating its average conductivity at each iteration only resulted in minor changes. This suggests that using the fixed value of $5 \Omega \mathrm{m}$ did not cause significant artifacts in deeper parts of the model.

\section{Regularization}

The results shown in the previous sections were obtained using smoothing regularization (i.e., $\mathbf{K}=\mathbf{L}^{T} \mathbf{L}$ in equation 4 ). To investigate the influence of regularization on the inversion and possible bias in the solution, we apply other regularization techniques. Using Krylov subspace methods to solve equation 4 for model updates introduces implicit regularization effects (Haber, 1997). During the solution of the normal equations, we successively build an approximation of the Krylov subspace spanned by the first $n_{\text {iter }}$ singular vectors of the Hessian matrix. By neglecting the remaining singular vectors, we regularize the solution (Hansen, 1998). Thus, we can control the amount of regularization by varying the number of CG iterations. To assess implicit regularization effects, we set the regularization parameter $\beta=0$ in equation 4 and compare inversion results obtained using increasing numbers of CG iterations in solving the normal equations.

Figure 9a-9d shows inversion results obtained using five, 25, 75, and 100 iterations to solve the normal equations without any explicit regularization. As anticipated, resolution and depth penetration improve with increasing number of CG iterations. The rms first decreases, but then it increases as the number of iterations increases from 75 to 100. Probably, at this point, noise present in the data starts gaining strong influence on the solution (Haber, 1997).

We also test a minimum-norm regularization (i.e., $\mathbf{K}=\mathbf{I}$ in equation 4). Setting $\gamma=10^{-4}$ in equation 6 and using $500 \mathrm{CG}$ iterations results in the image shown in Figure 9e. Because the additional stabilizing functional complements implicit regularization, using a large number of CG iterations here does not lead to a strongly noisy solution.

The models shown in Figures 8b, 9c, and 9e all explain the data fairly well, demonstrating the nonuniqueness of the inverse problem. All three models exhibit similar structures, indicating that
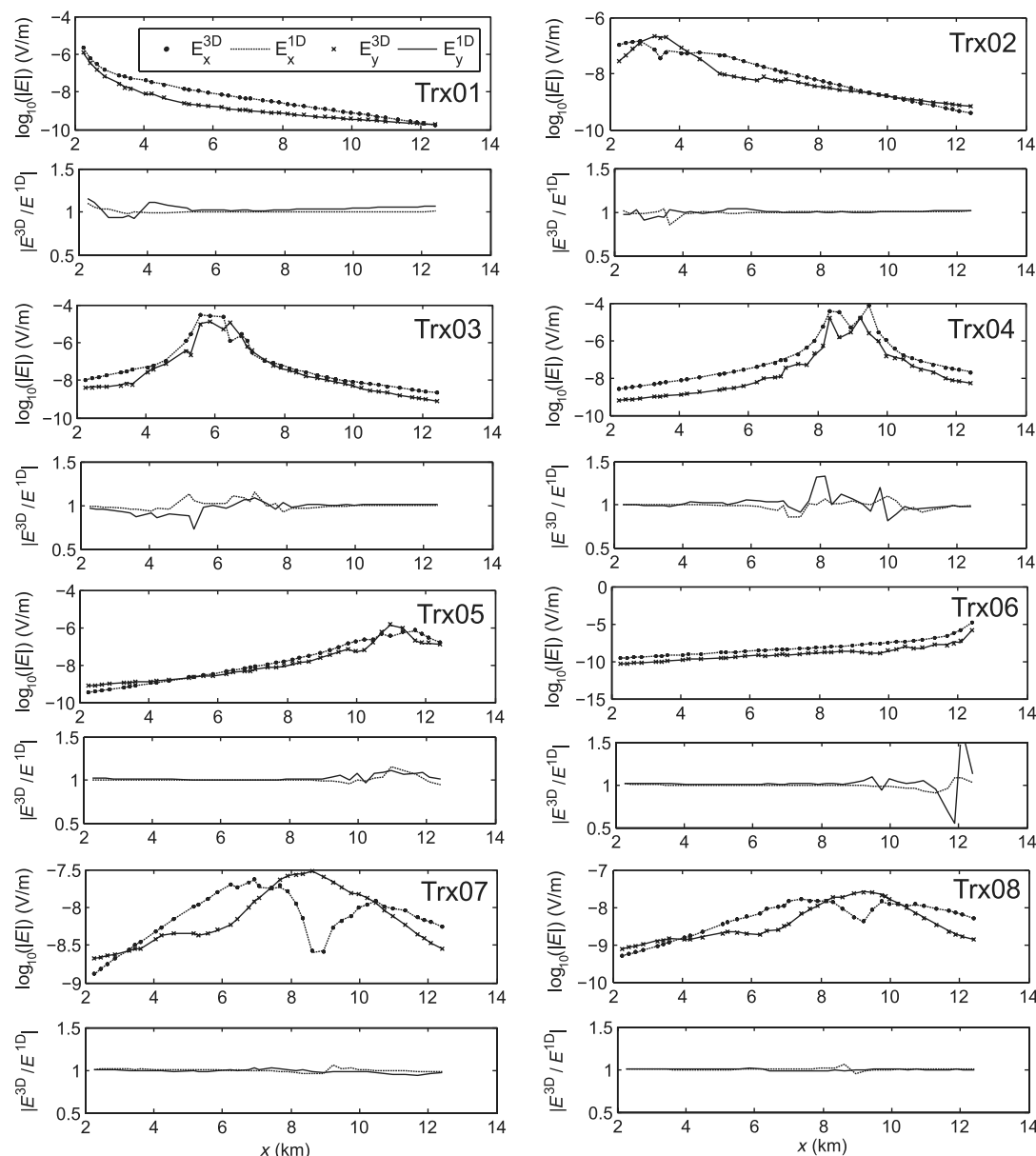

Figure 6. The 3D numerical (circles and crosses) and 1D quasianalytical (dashed and solid lines) solutions for $E_{x}$ and $E_{y}$ for the model shown in Figure 5a at a frequency of $0.98 \mathrm{~Hz}$. Each pair of plots shows field values and amplitude ratios for one transmitter. 
the main features in the images are robust and not artifacts of a particular regularization scheme. The solution obtained using smoothing regularization is more continuous, but results in a thicker conductor below the resistive layer at an approximately 1-km depth. This can be expected because the smoothing regularization minimizes the second spatial derivative of the model and thus favors smooth solutions. In contrast, for the minimum-norm and implicit regularizations, no such constraint is enforced. Accordingly, both images exhibit stronger variability, especially near the surface, than the smoothing-regularized image.

\section{Computational cost}

All inversions were run on a distributed platform consisting of four interconnected nodes, each equipped with two 12-core AMD Opteron 2.2 GHz CPUs and $64 \mathrm{~GB}$ of RAM. Each inversion used 64 MPI processes with 16 on each node.

The time and memory requirements are summarized in Table 1. The computation times shown are the averages of times measured throughout an inversion run. Running 30 inversion iterations and producing one of the images shown in the previous sections took approximately three days. The time spent on forward modeling and

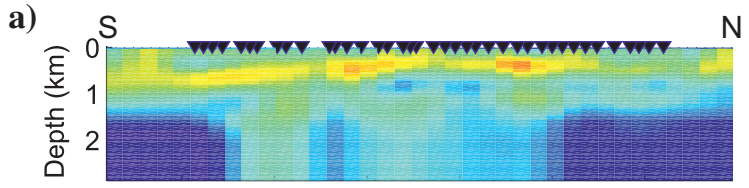

b)

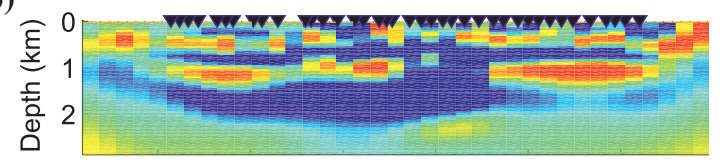

c)

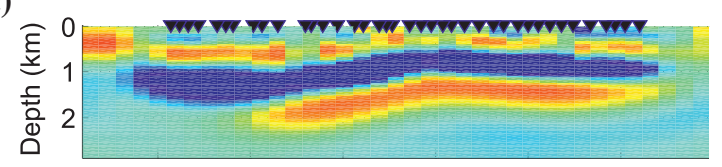

d)

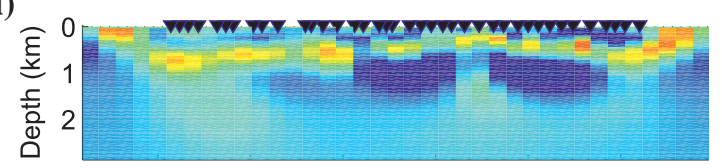

e)

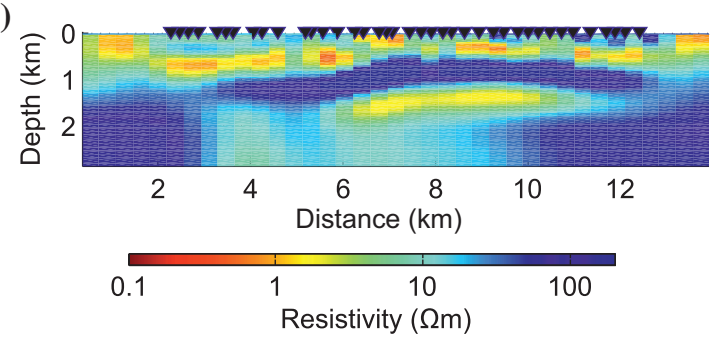

Figure 7. (a) Resistivity section along the profile line marked in Figure 1 derived from volume interpolation of $1 \mathrm{D}$ inversion results. Sections extracted from 3D inversion results for homogeneous halfspace starting models of (b) $1 \Omega \mathrm{m}, \mathrm{rms}=12.9$, (c) $5 \Omega \mathrm{m}$, $\mathrm{rms}=13.5$, (d) $20 \Omega \mathrm{m}, \mathrm{rms}=23.3$, and (e) starting from the model shown in (a) $\mathrm{rms}=14.9$.
Jacobian matrix computations comprises more than $90 \%$ of the total time. The memory required for factorizing the forward operator amounts to $85 \%$ of the total memory allocation.

\section{A PREFERRED MODEL}

\section{Data fit}

Based on various parameter tests and the analysis presented, we choose the model shown in Figure $8 \mathrm{~b}$ as our preferred model. The development of the rms value for this model is shown in Figure 10a. After approximately 25 iterations, the rms remains nearly constant. Figure 10b shows histograms of the initial and final normalized residuals $\mathbf{r}=\mathbf{W}_{d}\left(\mathbf{f}(\mathbf{m})-\mathbf{d}^{\text {obs }}\right)$. Clearly, the residuals are significantly lower for the final model and are centered around zero. The latter indicates that there is no systematic mismatch in the responses produced by the final model. The distribution of the residuals has rather heavy tails and thus differs strongly from a standard normal distribution. This results in the overall high final rms. The rms value could be easily decreased by choosing a higher error floor. For example, by setting the error floor to $10 \%$, after 30 iterations, the inversion produced a similar model with an rms of 3.2.

a)

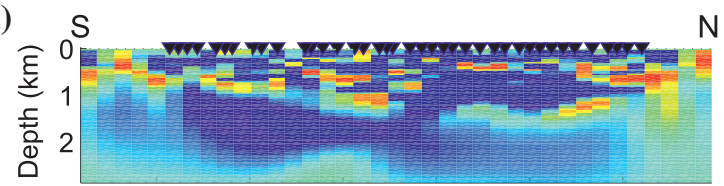

b)

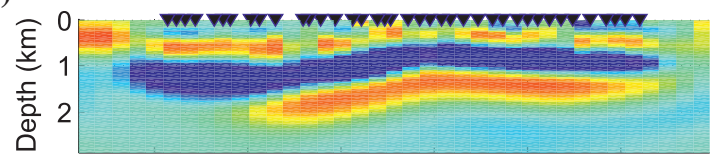

c)

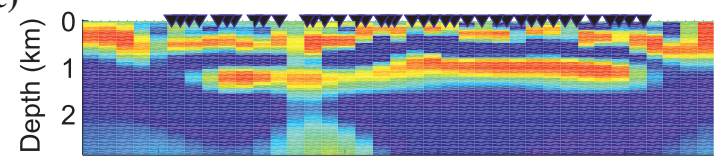

d)

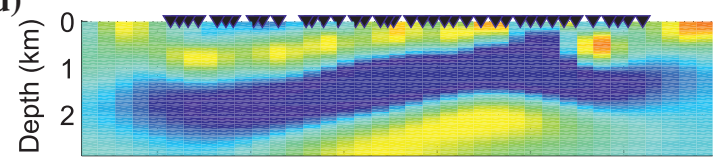

e)

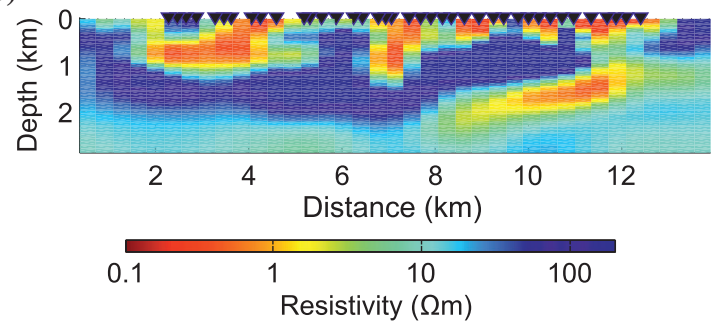

Figure 8. Images obtained by fixing the resistivity of the layer containing the sources at $5 \Omega \mathrm{m}$, with the thickness of that layer being: (a) $2 \mathrm{~m}, \mathrm{rms}=17.1$; (b) $5 \mathrm{~m}, \mathrm{rms}=13.5$; and (c) $40 \mathrm{~m}$, rms $=20.8$. Images obtained with the sources artificially shifted into the air to a height of (d) $1 \mathrm{~m}, \mathrm{rms}=24.5$ and (e) $15 \mathrm{~m}, \mathrm{rms}=116.5$. 

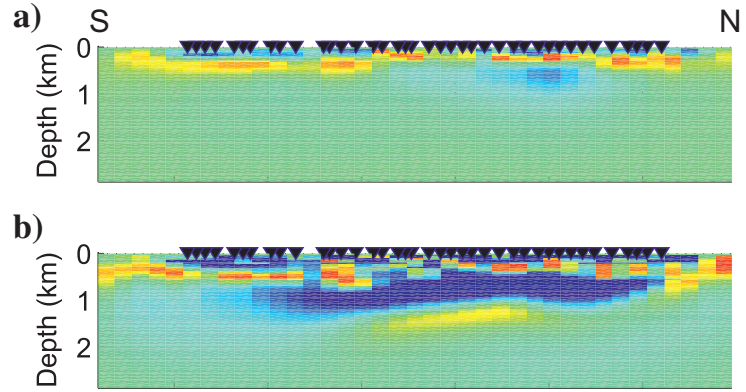

c)

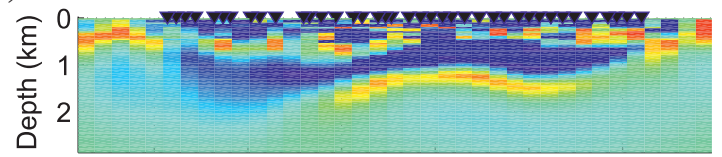

d)

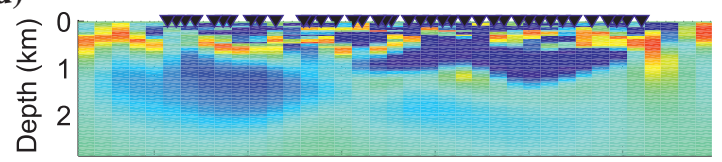

e)

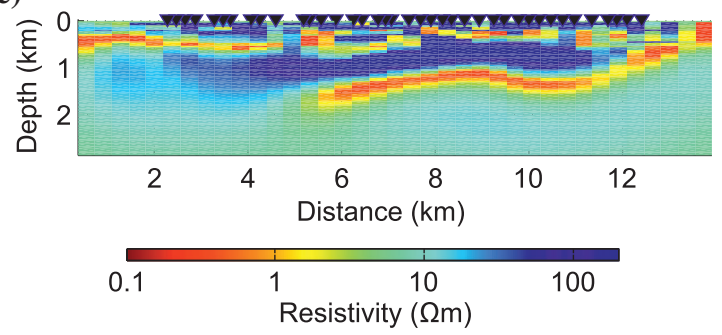

Figure 9. Images obtained without explicit regularization $(\beta=0$ in equation 1) and increasing numbers of CG iterations used for solving the normal equations: (a) 5 iterations, $\mathrm{rms}=20.3$; (b) 25 iterations, $\mathrm{rms}=13.5$; (c) 75 iterations, $\mathrm{rms}=13.0$; and (d) 100 iterations, rms $=14.5$. (e) The minimum-norm solution with $\gamma=10^{-4}$ in equation 6 and $500 \mathrm{CG}$ iterations, $\mathrm{rms}=12.9$.

Table 1. Average computation time and memory allocation for the real data inversion using four cluster nodes and 64 MPI processes.

Grid size

Number of unknowns in forward modeling

$50 \times 54 \times 80$

Number of unknowns in inversion

648,000

94,400

Time per inversion iteration

$2 \mathrm{~h} 30 \mathrm{~min}$

Factorization (number. required per inversion iteration)

Forward solution (number. required per inversion iteration)

Primary field calculation

$\min 50 \mathrm{~s}(5)$

0.6 s (4317)

Line search (including forward modeling)

Other operations (solution of model update equations, calculation of norms,

communication, output)

Total memory usage (GB)

$22 \min$

$40 \mathrm{~min}$

$35 \mathrm{~min}$

Factorization (GB)

Jacobian matrix (GB)
We expect that more faithful subsurface images are obtained if the best-determined data are given the highest weights. Therefore, we prefer using a low error floor of $2 \%$ and let the inversion use error estimates obtained from data processing for data values with uncertainties larger than $2 \%$.

The individual misfits for each receiver with respect to all frequencies, transmitters, and response functions are shown in Figure 10c for the initial and final iterations. We generally reduce the misfits six to seven times for all receivers except for those located in the center of the profile between $\approx 6$ and $8 \mathrm{~km}$. Larger misfits can be expected here because these data are most strongly affected by noise from a nearby transformer station and a gas pipeline carrying pulsed anticorrosion currents that crosses the receiver line at $\approx 7 \mathrm{~km}$. Many data points with large initial misfits that would likely have been excluded by manual data editing have been fitted reasonably well. This is evident, for example, from the reduction of the huge initial $\mathrm{rms}$ of one receiver at $6.5 \mathrm{~km}$ to an rms value within the range of the other receivers after inversion.

a)

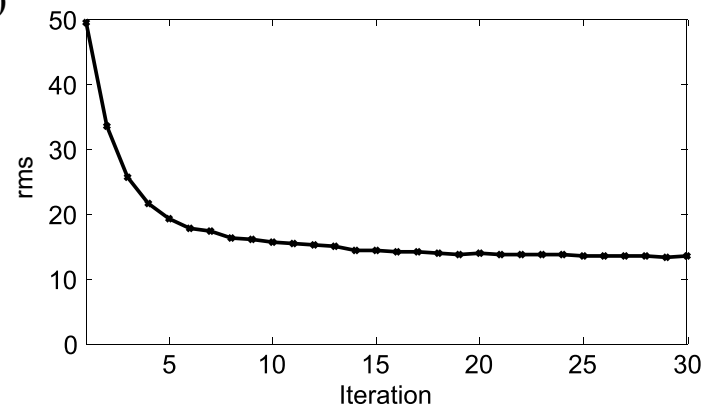

b)

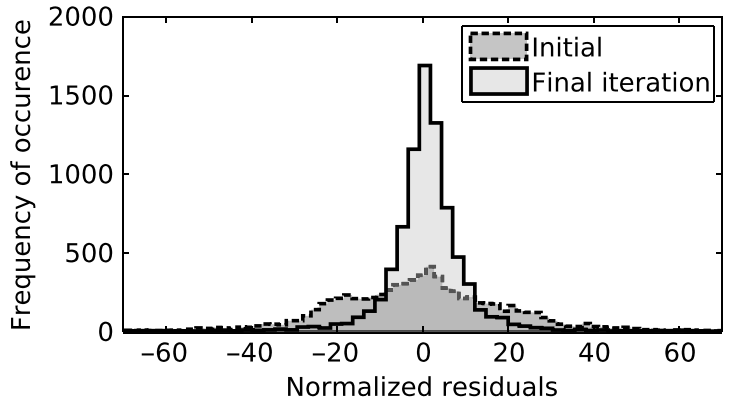

c)

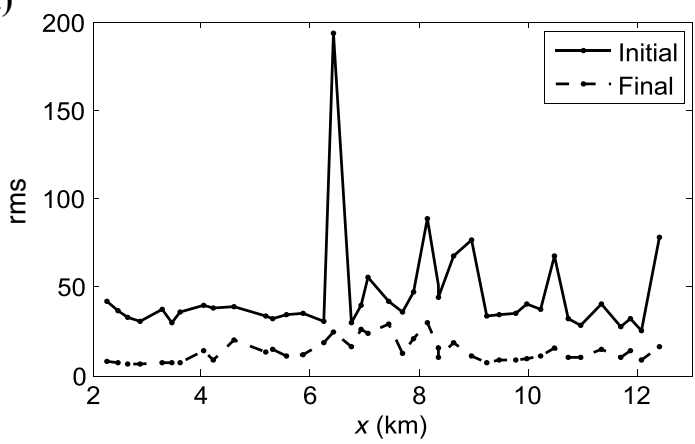

Figure 10. (a) The rms versus iteration count during the inversion for the model shown in Figure 8b. (b) Histograms of initial and final normalized residuals. (c) Initial and final data misfits for each receiver with respect to all frequencies, transmitters and response functions. 
We further analyze the data fit for individual points. Figure 11 shows representative examples of measured data, initial responses for the homogeneous half-space of $5 \Omega \mathrm{m}$, and final responses calculated for the model shown in Figure 8b. Most data points exhibit reasonable fit, with some exceptions, mainly at points where the measured data are distorted (e.g., transmitter Trx08 around $7.5 \mathrm{~km}$ ). Results similar to those shown are obtained for other frequencies, response functions and the $E_{y}$ component.

\section{Sensitivity and resolution analysis}

Because the Jacobian matrix is calculated explicitly at each iteration, we can readily analyze it. First, for convenient and compact visualization of the information contained in this matrix, we calculate cumulative sensitivity, as the $L_{2}$-norm of each column of the weighted sensitivity matrix. For every model parameter, this results in a single sensitivity value that includes sensitivities with respect to all data values. Squared cumulative sensitivities comprise the main diagonal of the approximate Hessian matrix (without the regularization term) in equation 4.

The normalized cumulative sensitivity distribution for the preferred model is shown in Figure 12. As expected, sensitivity is highest along the profile, near the sources and receivers. Remarkably, sensitivity is very high within the resistor at depths around $1 \mathrm{~km}$. This is consistent with other studies (e.g., Weiss and Constable, 2006) and can be explained by significant excitation of TMmode fields produced by the horizontal long-wire electric source.

Although cumulative sensitivity provides a general overview of coverage and is easy to calculate, it is a relative measure that does
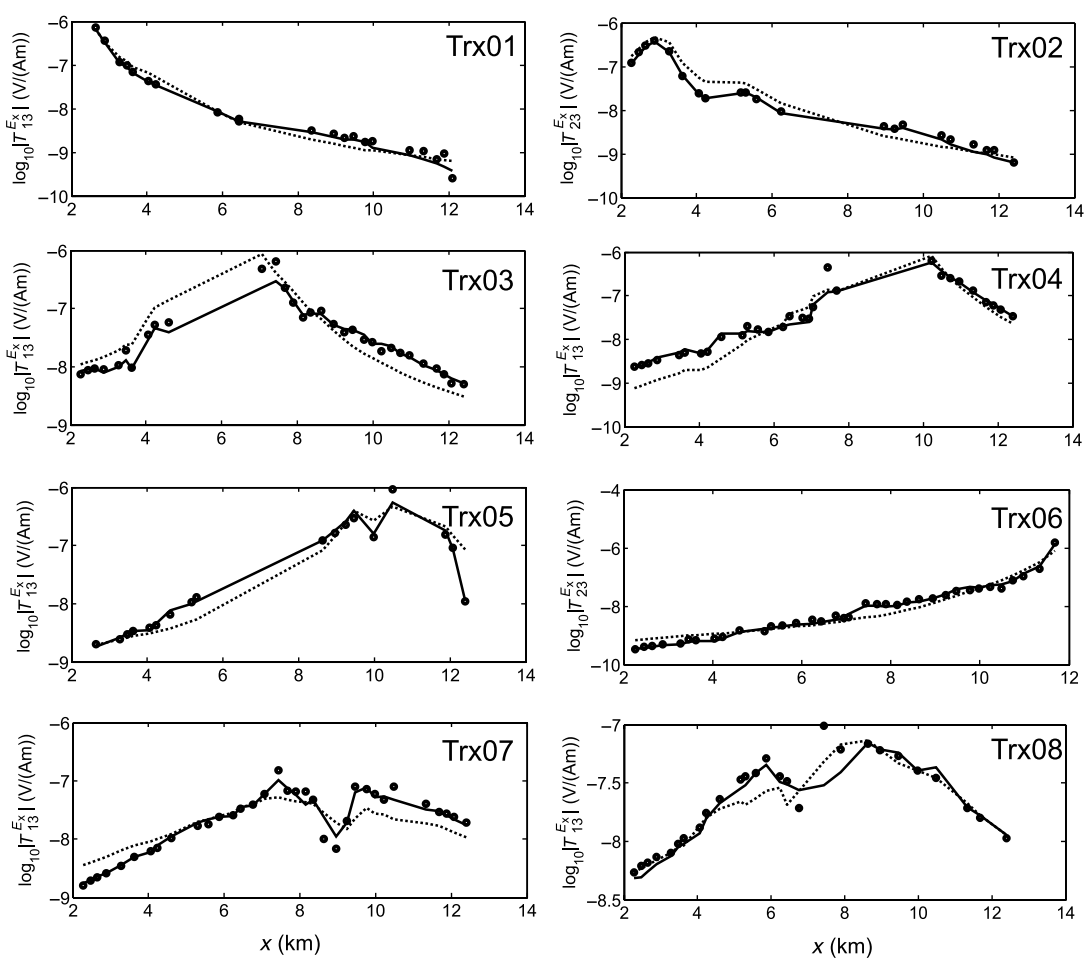

Figure 11. Amplitude of the $E_{x}$ response functions for a frequency of $0.4 \mathrm{~Hz}$ : observed data (circles), data for the initial model (dashed line), and data for the final model shown in Figure $8 \mathrm{~b}$ (solid line). Each plot represents data from one transmitter for all receivers along the survey line. not directly permit inferences about spatial resolution or depth penetration. Resolution and penetration can be assessed more thoroughly from other quantities that are based on singular-value decomposition (SVD) of the Jacobian. For consistency with the system of normal equation 4 , we analyze the matrix

$$
\hat{\mathbf{J}}=\left(\begin{array}{l}
\operatorname{Re}\{\overline{\mathbf{J}}\} \\
\operatorname{Im}\{\overline{\mathbf{J}}\}
\end{array}\right) \in \mathcal{R}^{2 N_{d} \times N_{m}} .
$$

The SVD of $\hat{\mathbf{J}}$ can be written as (Björck, 1996)

$$
\hat{\mathbf{J}}=\mathbf{U S V}^{T}=\left(\begin{array}{ll}
\mathbf{U}_{r} & \mathbf{U}_{0}
\end{array}\right)\left(\begin{array}{cc}
\mathbf{S}_{r} & 0 \\
0 & 0
\end{array}\right)\left(\begin{array}{c}
\mathbf{V}_{r}^{T} \\
\mathbf{V}_{0}^{T}
\end{array}\right)
$$

The singular values $s_{i}\left(i=1, \ldots, 2 N_{d}\right)$ on the main diagonal of $\mathbf{S} \in \mathcal{R}^{2 N_{d} \times N_{m}}$ are in decreasing order and comprise the spectrum of $\hat{\mathbf{J}}$. Matrices $\mathbf{U} \in \mathcal{R}^{2 N_{d} \times 2 N_{d}}$ and $\mathbf{V} \in \mathcal{R}^{N_{m} \times N_{m}}$ represent left and right singular vectors, respectively, and they are orthonormal. Matrices $\mathbf{S}_{r} \in \mathcal{R}^{r \times r}, \mathbf{U}_{r} \in \mathcal{R}^{r \times r}$, and $\mathbf{V}_{r}^{T} \in \mathcal{R}^{r \times N_{m}}$ with $r \leq 2 N_{d}$, denote the nonzero singular values and associated singular vectors.

The model resolution matrix (Menke, 1984) can be used to obtain quantitative estimates of resolution. To analyze how the subsurface is constrained by the data alone, we assume zero regularization. Then, the model resolution matrix is calculated using the right singular vectors obtained from SVD of $\hat{\mathbf{J}}$ as

$$
\mathbf{R}=\mathbf{V}_{r} \mathbf{V}_{r}^{T}
$$

Each row of this matrix is the PSF of the corresponding model parameter (Backus and Gilbert, 1968). Ideally, PSFs should be delta functions and $\mathbf{R}=\mathbf{I}$. For discrete inverse problems, PSFs describe averaging kernels. The conductivity of a model cell is then given as a weighted average of conductivities in the vicinity of the cell, and the width of the PSF is a measure of resolution (Menke, 1984).

Similar to Alumbaugh and Newman (2000), for each model parameter, we fold one row of the model resolution matrix into a $3 \mathrm{D}$ array and calculate the width at which the amplitude of the PSF is reduced by $50 \%$ along each dimension. Figure $13 \mathrm{a}$ and $13 \mathrm{c}$ shows the $50 \%$ PSF width in the $x$ - and $z$-directions for the preferred model using all $2 N_{d}=7914$ singular vectors. PSF widths are on the order of 300-400 $\mathrm{m}$ in the $x$-direction and close to $100 \mathrm{~m}$ in the vertical direction throughout large parts of the section. It is, however, too optimistic to include all singular vectors when estimating resolution length. A solution obtained for equation 4 using all singular vectors or, equivalently, $2 N_{d} \mathrm{CG}$ iterations will inherit a significant amount of noise. Furthermore, using $2 N_{d} \mathrm{CG}$ iterations and thus attempting to solve equation 4 exactly may deteriorate the inversion because equation 4 is only a linearized approximation to the solution of the full nonlinear problem. Introducing noise and overfitting are avoided by adding regularization to damp contributions from singular vectors 
corresponding to small singular values, or by decreasing the number of CG iterations. Therefore, we also show PSF widths for a resolution matrix with only the first 500 singular vectors included (Figure 13b and 13d). As expected, less information can be resolved from the reduced basis and resolution decreases significantly with depth. Similar conclusions were made for DC resistivity (Friedel, 2003) and potential field inversions (Fedi et al., 2005).

Assuming that the rank $r$ of the Jacobian matrix equals $2 N_{d}$ (i.e., all data values are linearly independent), the unbiased solution of the nonregularized linearized inverse problem is given by

$$
\delta m=\sum_{i=1}^{r} \frac{\mathbf{u}_{i}^{T} \delta \hat{\mathbf{d}}}{s_{i}} \mathbf{v}_{i},
$$

where

$$
\delta \hat{\mathbf{d}}=\left(\begin{array}{cc}
\mathbf{W}_{d} & 0 \\
0 & \mathbf{W}_{d}
\end{array}\right)\left(\begin{array}{c}
\operatorname{Re}\left[\mathbf{d}^{\mathrm{obs}}\right] \\
\operatorname{Im}\left[\mathbf{d}^{\text {obs }}\right]
\end{array}\right)
$$

The solution is thus a linear combination of the right singular vectors with coefficients proportional to the data residuals and the reciprocals of the singular values. By summing over the first $p<$ $r$ singular vectors, a truncated SVD (Jupp and Vozoff, 1975; Pedersen, 2004) is realized.

To analyze depth penetration, and study how the number of singular vectors included in the solution of the normal equations influences penetration depth, we use depth-resolution plots (Fedi et al., 2005). Such plots are obtained by folding every right singular vector into a $3 \mathrm{D}$ array and calculating the $L_{2}$-norms of the values over each horizontal slice. For every right singular vector, we obtain $N_{z}$ values corresponding to the vertical model discretization. Plotting these values for every singular vector gives an overview of the relative contribution of every right singular vector to each depth level.

A depth-resolution plot of the Jacobian matrix calculated for the preferred model is shown in Figure 14. Low values at depths larger than $500 \mathrm{~m}$ for the first $\approx 100$ vectors indicate that these vectors contain little information on deeper parts of the model. This is consistent with the observation that more $\mathrm{CG}$ iterations used for solving the normal equations result in better imaging of deep structures (see Figure 9).

Because the model resolution matrix given by equation 13 is calculated for a particular Jacobian matrix, it represents an analysis of the linearized problem rather than the full nonlinear inverse problem (Pedersen, 2004; Kalscheuer and Pedersen, 2007). Linearized analysis is likely to give pessimistic resolution estimates, although the results should be qualitatively similar to those obtained for a nonlinear analysis (Kalscheuer et al., 2010).

The analysis presented may seem excessively expensive. Indeed, a full SVD in equation 12 would require memory of $O\left(N_{m}^{2}\right)$. However, we are not interested in the singular vectors that span the null space of the Jacobian. Therefore, we calculate the compact $\mathrm{SVD} \hat{\mathbf{J}}=\mathbf{U}_{r} \mathbf{S}_{r} \mathbf{V}_{r}^{T}$, which requires memory of $O\left(N_{d} N_{m}\right)$ and is relatively fast because $2 N_{d} \ll N_{m}$. Using a new distributed library for dense linear algebra (Poulson et al., 2012) and 32 cores, it took approximately five minutes and only 30 GB of memory to calculate the compact SVD of the $7194 \times 94,400$ double-precision real Jacobian matrix. Finally, to obtain PSF widths, the model resolution matrix $\mathbf{R} \in \mathcal{R}^{N_{m} \times N_{m}}$ is

a)

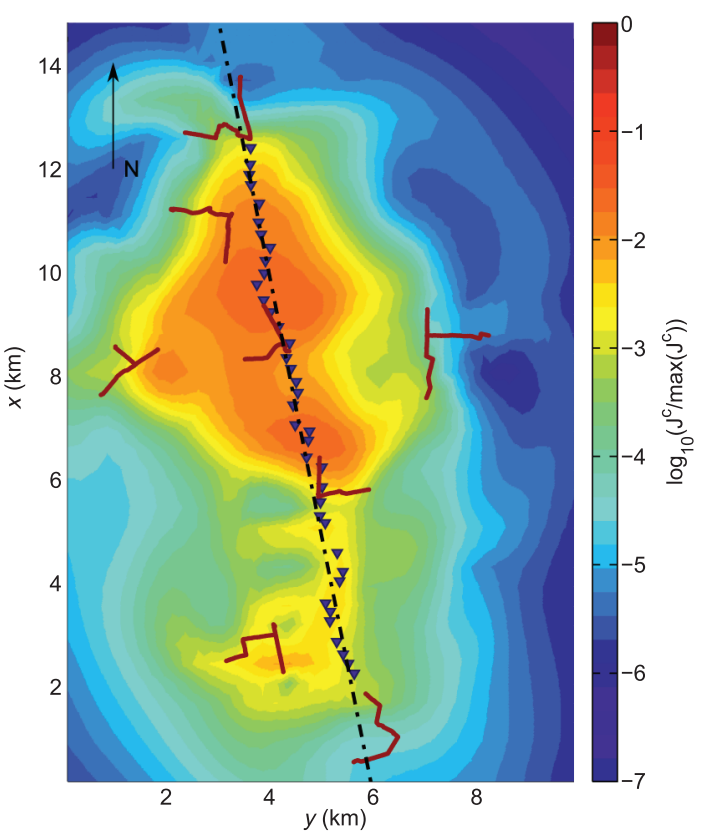

b) $\mathrm{s}$

$\mathrm{N}$

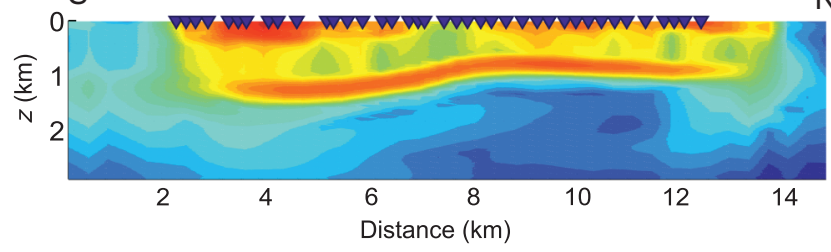

Figure 12. Logarithm of normalized cumulative sensitivity computed for the final inversion model (see Figure $8 \mathrm{~b}$ ) and the data subset used for the inversion. (a) Horizontal section at $z=0.8 \mathrm{~km}$ and (b) vertical section along the line depicted in (a). a)

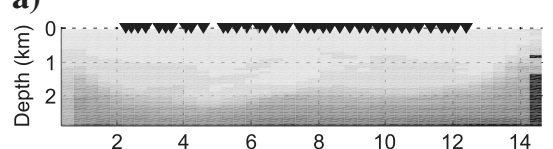

b)

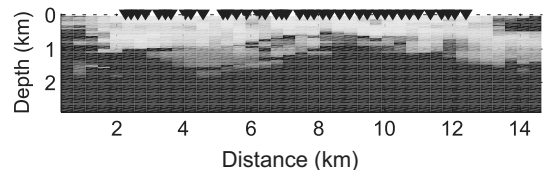

c)

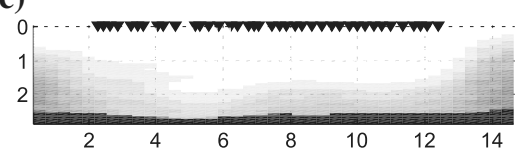

d)

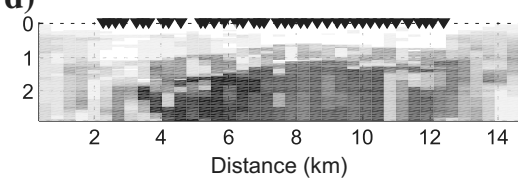

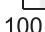

1000

$50 \%$ Spread width (m)

Figure 13. The 50\% PSF width for the model shown in Figure $8 \mathrm{~b}$ and the field acquisition geometry. PSFs were calculated (a) in the $x$-direction using the full basis of 7914 singular vectors, (b) in the $x$-direction using a reduced basis of 500 singular vectors, (c) in the $z$-direction using the full basis, and (d) in the $z$-direction using a reduced basis of 500 singular vectors. 


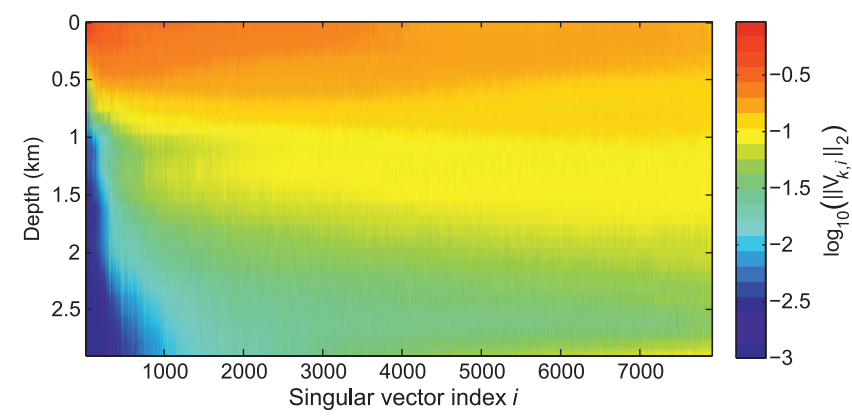

Figure 14. Depth-resolution plot for the Jacobian matrix calculated using the preferred model (see Figure $8 \mathrm{~b}$ ) and the field acquisition geometry. Each column represents the $L_{2}$-norms for depth slices $k=1 \ldots N_{z}$, calculated for $i=1 \ldots 2 N_{d}$ singular vectors of the Jacobian matrix.

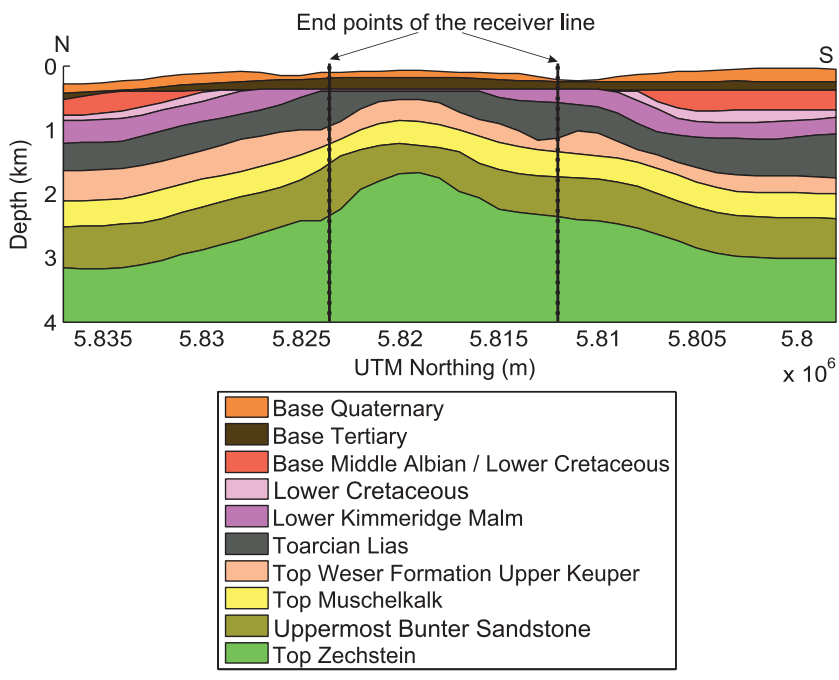

Figure 15. Section extracted from the regional structural geologic model by Klapperer et al. (2011) along the CSEM profile.

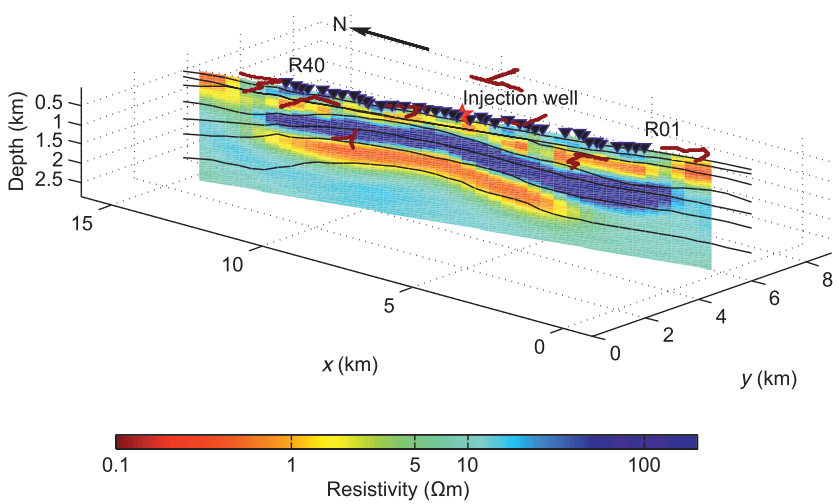

Figure 16. Section extracted from the preferred 3D inversion model with structural horizons from Figure 15 overlain. The red lines and black triangles depict transmitters and receivers, respectively. The red star denotes the $\mathrm{CO}_{2}$ injection well. The starting model was a homogeneous half-space of $5 \Omega \mathrm{m}$. not formed explicitly. Instead, the PSF for the $i$ th model parameter is computed as

$$
\mathbf{r}_{i}=\mathbf{V}_{r}\left(\mathbf{V}_{r}^{T} \mathbf{e}\right), \quad i=1 \ldots N_{m},
$$

where the vector $\mathbf{e}$ has only one nonzero element $\mathbf{e}_{i}=1$. After estimating the widths for three directions, the calculated PSF is discarded immediately. The procedure is then repeated for each model parameter. Therefore, the computational and memory complexities of the PSF widths calculation are nearly equal to those of the compact SVD of $\hat{\mathbf{J}}$.

\section{Interpretation}

The model shown in Figure 8b contains several prominent conductive and resistive laterally continuous structures. The regional geology is known to comprise an anticline structure of sediments overlying a salt pillow (Förster et al., 2009). Figure 15 shows a slice extracted from a regional structural geologic model of the Ketzin region (Klapperer et al., 2011) along the CSEM profile. This structural model was derived using several seismic profiles and boreholes. The stratigraphic division is mainly based on information provided in Stackebrandt and Manhenke (2004). This geologic model was not used as a priori information for the CSEM inversion.

To compare the structural horizons of this geologic model to the CSEM inversion result, we overlay the two models in Figure 16. The structures resolved by the CSEM inversion correlate well with the geologic horizons, generally following the anticline trend for all layers and depths, except for regions near the ends of the survey line that the CSEM data are not sensitive to. We will provide a rough geologic interpretation per stratum, starting from the deepest and oldest unit.

The section consists of a series of sedimentary layers combined into larger units and groups. The base is formed by a Zechstein unit, which is primarily rock salt. The resolution and sensitivity studies presented earlier indicate that we have very low sensitivity to this deep unit that is located at depths below $2 \mathrm{~km}$ along the CSEM profile. Nevertheless, the deviations from the starting model in this region are significant, especially near the top of the anticline. We interpret the resistive structure indicated here as an imprint of the salt pillow. The Zechstein is overlain by a Bunter unit consisting of sandstone layers. The Bunter sandstone unit contains many saline aquifers. This can explain the low resistivity observed at depths of $1-1.5 \mathrm{~km}$. The prominent resistive layer above the good conductor is likely related to Muschelkalk and Keuper stratigraphic units. These units comprise sequences of limestone, dolostone, gypsum, claystone, and anhydrite beds, which are likely responsible for overall high resistivity. These units are similar in composition and thus can be expected to have similar resistivities; likely, CSEM resolution is not sufficient to distinguish between them. The conductive layer on top of the prominent resistor is probably related to the Lias Group containing several regional saline aquifers. Finally, the resistive near-surface layer represents weathered Tertiary and Quaternary sediments.

\section{CONCLUSIONS}

We have successfully applied 3D inversion to land-based CSEM data from the Ketzin $\mathrm{CO}_{2}$ storage formation. A novel three-phase transmitter configuration has been instrumental for acquiring data 
of sufficient quality. Uncertainty estimates from data processing have been usable for data preselection and weighting within the inversion. Data analysis and synthetic studies indicated redundancy of the magnetic field components for this survey and further redundancy in the response functions and frequency components extracted from the field data. Data preselection aimed at reducing redundancy has been useful for stabilizing and accelerating the inversion, thus allowing us to run extensive tests for validating the inversion model. For future land CSEM surveys of similar targets, survey efficiency may be enhanced by acquiring electric field data only; however, magnetic field data are required if additional magnetotelluric evaluation is intended.

Inversion grid analysis and accuracy checks of the modeled responses confirmed that a secondary-field approach can accurately model EM fields for our complex long-wire source geometries on a relatively coarse grid. The requirement to have equal background and $3 \mathrm{D}$ conductivities at the source locations is a drawback of the secondary-field approach. Our strategy of fixing conductivity within a thin layer containing the sources has been a viable work-around for the Ketzin site. For sites with significant topography, other strategies may be required, such as homogenizing conductivity only for the small groups of cells containing one of the sources and using different background models for different sources.

Analysis of inversion results for various starting models and regularizations increases confidence in our results. Smoothing and minimum-norm regularization operators, and implicit regularization resulting from the incomplete iterative solution of the normal equations, all lead to model characteristics consistent with theoretical expectations. The derived models are similar and explain the data similarly well, confirming that the resolved structures are not artifacts of a particular regularization technique.

Because we calculate the Jacobian matrix explicitly, we can carry out sensitivity and resolution analysis at marginal additional cost. Cumulative sensitivity is easy to calculate and provides insights about survey coverage. For our survey, cumulative sensitivity suggests comparatively high confidence in a prominent resistor at approximately 1-km depth and lower confidence in the underlying as well as the overlying conductor. More detailed, quantitative analysis of spatial resolution and depth penetration can be achieved by assessing PSF widths and depth-resolution plots, respectively. Because both measures are derived from truncated SVD of the (nonregularized) approximate Hessian, they are closely related to the number of CG iterations used for solving the system of normal equations. Resolution length estimates for our preferred inversion model are broadly consistent with the scale of features resolved in the model. Resolution lengths may have been somewhat overestimated; the analysis presented can be refined to take into account the nonlinearity of the inversion problem and to estimate model uncertainties. A depth-resolution plot for the preferred model suggests that the 500 CG iterations used do not fully exploit the depth information potentially contained in the data. Nevertheless, it is unlikely that using more CG iterations would increase depth penetration because the required additional regularization effectively damps contributions from the small singular values associated with further iterations.

Using distributed computations extensively, it is feasible to run the direct-solver-based 3D Gauss-Newton inversion for a field data set and carry out resolution analysis in reasonable times on moderate-size computational platforms that are now widely available.
Without considering any geologic a priori information, starting from a homogeneous half-space, the Gauss-Newton minimization scheme has achieved good data fit and provides a resistivity model that agrees well with the known geologic structures. The results can potentially be improved in the future by applying structurally constrained inversion.

\section{ACKNOWLEDGMENTS}

We acknowledge N. Meqbel and M. Becken for many useful discussions on practical aspects of inversion. Comments by three anonymous reviewers helped improve the manuscript. This work is part of the MULTI-EM project funded by the German Ministry of Education and Research, grant no. 03G0746A-MULTI-EM. The Ketzin data set was collected within the GeoEn project with the invaluable help of the GFZ Potsdam EM research group and students. The receiver instruments were provided by the Geophysical Instrument Pool Potsdam (GIPP).

\section{REFERENCES}

Alumbaugh, D. L., and G. A. Newman, 2000, Image appraisal for 2-D and 3-D electromagnetic inversion: Geophysics, 65, 1455-1467, doi: 10 $.1190 / 1.1444834$.

Amestoy, P. R., A. Guermouche, J.-Y. L'Excellent, and S. Pralet, 2006, Hybrid scheduling for the parallel solution of linear systems: Parallel Computing, 32, 136-156, doi: 10.1016/j.parco.2005.07.004.

Backus, G., and F. Gilbert, 1968, The resolving power of gross Earth data: Geophysical Journal of the Royal Astronomical Society, 16, 169-205, doi: 10.1111/j.1365-246X.1968.tb00216.x.

Balay, S., J. Brown, K. Buschelman, V. Eijkhout, W. D. Gropp, D. Kaushik, M. G. Knepley, L. C. McInnes, B. F. Smith, and H. Zhang, 2012, PETSc users manual: Technical Report ANL-95/11-Revision 3.3, Argonne National Laboratory.

Björck, A., 1996, Numerical methods for least squares problems: SIAM.

Chave, A. D., 2009, On the electromagnetic fields produced by marine frequency domain controlled sources: Geophysical Journal International, 179, 1429-1457, doi: 10.1111/j.1365-246X.2009.04367.x.

Commer, M., S. L. Helwig, A. Hördt, and B. Tezkan, 2005, Interpretation of long-offset transient electromagnetic data from Mount Merapi, Indonesia using a three-dimensional optimization approach: Journal of Geophysical Research, 110, B03207, doi: 10.1029/2004JB003206.

Commer, M., and G. Newman, 2008, New advances in three-dimensional controlled-source electromagnetic inversion: Geophysical Journal International, 172, 513-535, doi: 10.1111/j.1365-246X.2007.03663.x.

Connell, D., and K. Key, 2013, A numerical comparison of time and frequency-domain marine electromagnetic methods for hydrocarbon exploration in shallow water: Geophysical Prospecting, 61, 187-199, doi: 10 $.1111 / \mathrm{j} .1365-2478.2012 .01037 . x$.

Constable, S., 2010, Ten years of marine CSEM for hydrocarbon exploration: Geophysics, 75, no. 5, A67-A81, doi: 10.1190/1.3483451.

Constable, S., 2013, Review paper: Instrumentation for marine magnetotelluric and controlled source electromagnetic sounding: Geophysical Prospecting, 61, 505-532, doi: 10.1111/j.1365-2478.2012.01117.x.

Cressie, N., 1993, Statistics for spatial data: John Wiley and Sons.

Fedi, M., P. C. Hansen, and V. Paoletti, 2005, Analysis of depth resolution in potential-field inversion: Geophysics, 70, no. 6, A1-A11, doi: 10.1190/1 .2122408 .

Förster, A., R. Giese, C. Juhlin, B. Norden, and N. Springer, 2009, The geology of the CO2SINK site: From regional scale to laboratory scale: Energy Procedia, 1, 2911-2918, doi: 10.1016/j.egypro.2009.02.066.

Förster, A., B. Norden, K. Zinck-Jørgensen, P. Frykman, J. Kulenkampff, E. Spangenberg, J. Erzinger, M. Zimmer, J. Kopp, G. Borm, C. Juhlin, C.-G Cosma, and S. Hurter, 2006, Baseline characterization of the CO2SINK geological storage site at Ketzin, Germany: Environmental Geosciences, 13, 145-161, doi: 10.1306/eg.02080605016.

Friedel, S., 2003, Resolution, stability and efficiency of resistivity tomography estimated from a generalized inverse approach: Geophysical Journal International, 153, 305-316, doi: 10.1046/j.1365-246X.2003 .01890.x.

Golub, G. H., and C. F. van Loan, 1996, Matrix computations, 3rd ed.: The Johns Hopkins University Press. 
Grayver, A. V., 2013, Three-dimensional controlled-source electromagnetic inversion using modern computational concepts: Ph.D. thesis, Free University of Berlin.

Grayver, A. V., R. Streich, and O. Ritter, 2013, Three-dimensional parallel distributed inversion of CSEM data using a direct forward solver: Geophysical Journal International, 193, 1432-1446, doi: 10.1093/gji/ ggt055.

Haber, E., 1997, Numerical strategies for the solution of inverse problems: Ph.D. thesis, The University of British Columbia.

Haber, E., U. Ascher, and D. Oldenburg, 2000, On optimization techniques for solving non-linear inverse problems: Inverse Problems, 16, 1263 1280, doi: 10.1088/0266-5611/16/5/309.

Hansen, P. C., 1998, Rank-deficient and discrete ill-posed problems: Numerical aspects of linear inversion: SIAM.

Hördt, A., P. Andrieux, F. M. Neubauer, H. Rüter, and K. Vozoff, 2000, A first attempt at monitoring underground gas storage by means of timelapse multichannel transient electromagnetics: Geophysical Prospecting, 48, 489-509, doi: 10.1046/j.1365-2478.2000.00192.x.

Jupp, D. L. B., and K. Vozoff, 1975, Stable iterative methods for the inversion of geophysical data: Geophysical Journal of the Royal Astronomical Society, 42, 957-976, doi: 10.1111/j.1365-246X.1975.tb06461.x.

Kalscheuer, T., M. Commer, S. L. Helwig, A. Hördt, and B. Tezkan, 2007, Electromagnetic evidence for an ancient avalanche caldera rim on the south flank of Mount Merapi, Indonesia: Journal of Volcanology and Geothermal Research, 162, 81-97, doi: 10.1016/j.jvolgeores.2006 .12.014.

Kalscheuer, T., M. de los Ángeles García Juanatey, N. Meqbel, and L. B. Pedersen, 2010, Non-linear model error and resolution properties from two-dimensional single and joint inversions of direct current resistivity and radiomagnetotelluric data: Geophysical Journal International, 182, 1174-1188, doi: 10.1111/j.1365-246X.2010.04686.x.

Kalscheuer, T., and L. B. Pedersen, 2007, A non-linear truncated SVD variance and resolution analysis of two-dimensional magnetotelluric models: Geophysical Journal International, 169, 435-447, doi: 10.1111/j.1365246X.2006.03320.x.

Keller, G. V., J. I. Pritchard, J. J. Jacobson, and N. Harthill, 1984, Megasource time-domain electromagnetic sounding methods: Geophysics, 49, 993-1009, doi: 10.1190/1.1441743.

Kim, J. H., and Y. Kim, 2011, A unified transformation function for lower and upper bounding constraints on model parameters in electrical and electromagnetic inversion: Journal of Geophysics and Engineering, 8, 21-26, doi: 10.1088/1742-2132/8/1/004

Klapperer, S., I. Moeck, and B. Norden, 2011, Regional 3D geological modeling and stress field analysis at the $\mathrm{CO}_{2}$ storage site of Ketzin, Germany: Geothermal Resources Council Transactions, 35, 419-424.

Kurtz, R. D., J. C. Macnae, and G. F. West, 1989, A controlled-source, timedomain electromagnetic survey over an upthrust section of Archean crust in the Kapuskasing Structural Zone: Geophysical Journal International, 99, 195-204, doi: 10.1111/j.1365-246X.1989.tb02024.X.

Li, M., A. Abubakar, J. Liu, G. Pan, and T. M. Habashy, 2011, A compressed implicit Jacobian scheme for 3D electromagnetic data inversion: Geophysics, 76, no. 3, F173-F183, doi: 10.1190/1.3569482.

Menke, W., 1984, Geophysical data analysis: Discrete inverse theory: Academic Press.

Minsley, B. J., B. D. Smith, R. Hammack, J. I. Sams, and G. Veloski, 2012, Calibration and filtering strategies for frequency domain electromagnetic data: Journal of Applied Geophysics, 80, 56-66, doi: 10.1016/j.jappgeo 2012.01.008

Mitsuhata, Y., T. Uchida, K. Matsuo, A. Marui, and K. Kusunose, 2006, Various-scale electromagnetic investigations of high-salinity zones in a coastal plain: Geophysics, 71, no. 6, B167-B173, doi: 10.1190/1 .2335658 .

Newman, G. A., and D. L. Alumbaugh, 1995, Frequency-domain modelling of airborne electromagnetic responses using staggered finite differences: Geophysical Prospecting, 43, 1021-1042, doi: 10.1111/j.1365-2478.1995 .tb00294.x.

Newman, G. A., M. Commer, and J. J. Carazzone, 2010, Imaging CSEM data in the presence of electrical anisotropy: Geophysics, 75, no. 2, F51-F61, doi: 10.1190/1.3295883.

Nocedal, J., and S. J. Wright, 1999, Numerical optimization: Springer
Oldenburg, D., E. Haber, and R. Shekhtman, 2013, Three dimensional inversion of multisource time domain electromagnetic data: Geophysics, 78, no. 1, E47-E57, doi: 10.1190/geo2012-0131.1.

Súilleabháin, L. O., M. Rosenquist, R.-E. Plessix, P. van Rensbergen, and D. Sebayang, 2012, Anisotropic inversion of CSEM data from offshore Malaysia: 82nd Annual International Meeting, SEG, Expanded Abstracts, doi: 10.1190/segam2012-0726.1.

Pedersen, L. B., 2004, Determination of the regularization level of truncated singular-value decomposition inversion: The case of $1 \mathrm{D}$ inversion of MT data: Geophysical Prospecting, 52, 261-270, doi: 10.1111/j.1365-2478 .2004.00414.X.

Pérez-Flores, M. A., R. G. Antonio-Carpio, E. Gómez-Treviño, I. Ferguson, and S. Méndez-Delgado, 2012, Imaging of 3D electromagnetic data at low-induction numbers: Geophysics, 77, no. 4, WB47-WB57, doi: 10 .1190/geo2011-0368.1.

Poulson, J., B. Marker, R. A. van de Geijn, J. R. Hammond, and N. A. Romero, 2012, Elemental: A new framework for distributed memory dense matrix computations: ACM Transactions on Mathematical Software, 39 , 13, doi: 10.1145/2427023.2427030.

Sasaki, Y., and M. A. Meju, 2009, Useful characteristics of shallow and deep marine CSEM responses inferred from 3D finite-difference modeling: Geophysics, 74, no. 5, F67-F76, doi: 10.1190/1.3168616.

Schwalenberg, K., M. Haeckel, J. Poort, and M. Jegen, 2010, Evaluation of gas hydrate deposits in an active seep area using marine controlled source electromagnetics: Results from Opouawe Bank, Hikurangi Margin, New Zealand: Marine Geology, 272, 79-88, doi: 10.1016/j.margeo.2009.07 006 .

Schwarzbach, C., and E. Haber, 2013, Finite element based inversion for time-harmonic electromagnetic problems: Geophysical Journal International, 193, 615-634, doi: 10.1093/gji/got006.

Siemon, B., A. V. Christiansen, and E. Auken, 2009, A review of helicopterborne electromagnetic methods for groundwater exploration: Near Surface Geophysics, 7, 629-646, doi: 10.3997/1873-0604.2009043.

Stackebrandt, W., and V. Manhenke, 2004, Atlas zur Geologie von Brandenburg: Landesamt für Geowissenschaften und Rohstoffe Brandenburg.

Streich, R., 2009, 3D finite-difference frequency-domain modeling of controlled-source electromagnetic data: Direct solution and optimization for high accuracy: Geophysics, 74, no. 5, F95-F105, doi: 10.1190/1 .3196241 .

Streich, R., and M. Becken, 2011, Electromagnetic fields generated by finite-length wire sources: Comparison with point dipole solutions: Geophysical Prospecting, 59, 361-374, doi: 10.1111/j.1365-2478.2010 .00926.x.

Streich, R., M. Becken, and O. Ritter, 2013, Robust processing of noisy land-based controlled-source electromagnetic data: Geophysics, $\mathbf{7 8}$ no. 5, E237-E247, doi: 10.1190/geo2013-0026.1.

Tikhonov, A. N., and V. Y. Arsenin, 1977, Solutions of ill-posed problems: Wiley.

Um, E. S., and D. L. Alumbaugh, 2007, On the physics of the marine controlled-source electromagnetic method: Geophysics, 72, no. 2, WA13WA26, doi: 10.1190/1.2432482

Weiss, C. J., and S. Constable, 2006, Mapping thin resistors and hydrocarbons with marine EM methods, Part II - Modeling and analysis in 3D Geophysics, 71, no. 6, G321-G332, doi: 10.1190/1.2356908.

Weitemeyer, K., G. Gao, S. Constable, and D. Alumbaugh, 2010, The practical application of 2D inversion to marine controlled-source electromagnetic data: Geophysics, 75, no. 6, F199-F211, doi: 10.1190/1.3506004

Weitemeyer, K. A., S. Constable, and A. M. Trehu, 2011, A marine electromagnetic survey to detect gas hydrate at Hydrate Ridge, Oregon: Geophysical Journal International, 187, 45-62, doi: 10.1111/j.1365246X.2011.05105.x.

Wirianto, M., W. A. Mulder, and E. C. Slob, 2010, A feasibility study of land CSEM reservoir monitoring in a complex 3-D model: Geophysical Journal International, 181, 741-755, doi: 10.1111/j.1365-246X.2010 .04544.x.

Yang, D., and D. W. Oldenburg, 2012, Three-dimensional inversion of airborne time-domain electromagnetic data with applications to a porphyry deposit: Geophysics, 77, no. 2, B23-B34, doi: 10.1190/geo2011-0194.1. Zhdanov, M. S., 2002, Geophysical inverse theory and regularization problems: Elsevier Science. 